\title{
Linearizing a Non-linear Formulation for General Relativistic Dissipative Fluids
}

\author{
T. Celora* and N. Andersson \\ Mathematical Sciences and STAG Research Centre, \\ University of Southampton, Southampton SO17 1BJ, UK \\ G.L. Comer \\ Department of Physics, Saint Louis University, St. Louis, MO 63156-0907, USA
}

(Dated: August 4, 2020)

\begin{abstract}
Fully non-linear equations of motion for dissipative general relativistic fluids can be obtained from an action principle involving the explicit use of lower dimensional matter spaces. More traditional strategies for incorporating dissipation — like the famous Müller-Israel-Stewart model —are based on expansions away from equilibrium defined, in part, by the laws of thermodynamics. The goal here is to build a formalism to facilitate comparison of the action-based results with those based on the traditional approach. The first step of the process is to use the action-based approach itself to build self-consistent notions of equilibrium. Next, first-order deviations are developed directly on the matter spaces, which motivates the latter as the natural arena for the underlying thermodynamics. Finally, we identify the dissipation terms of the action-based model with firstorder "thermodynamic" fluxes, on which the traditional models are built. A simple application of a single viscous fluid is considered. The description is developed in a general setting so that the formalism can be used to describe more complicated systems, for which causal and stable models are not yet available. Finally, even though our expansions are halted at first order, we sketch out how a causal response can be implemented with telegraph-type equations.
\end{abstract}

\footnotetext{
*T.Celora@soton.ac.uk
} 


\section{INTRODUCTION}

The covariant nature of general relativity highlights the central role played by the reference frame used to describe a physical system. At the same time, the evolution of dissipative fluids must be consistent with thermodynamic principles and the arrow of time associated with the second law. Matching these two pictures - general relativity and thermodynamicsposes interesting foundational questions, and therefore, it is not surprising that the construction of general relativistic models for dissipative fluids constitutes a problem that has kept physicists busy for a long time.

Early attempts, like the seminal work of Eckart [1] and Landau and Lifshitz [2], date back to the first half of the last century. These models are essentially the same and-even though the Landau-Lifschitz version is a little less pathological-have been proven to suffer from causality and stability shortcomings which cannot be ignored in a relativistic approach. Important steps forward were taken by Müeller [3] in the 1960s and Israel and Stewart in the 1970s [4-7]. Their results have been shown (Hiscock and Lindblom [8]) to resolve the stability and causality issues of the earlier attempts. However, a number of other issues remain to be addressed.

First, the Müller-Israel-Stewart (MIS) model is based on an implicit expansion in deviations away from thermal equilibrium, and has been demonstrated to fail when large deviations are considered (see Hiscock and Lindblom [9]). Second, from a field-theory perspective, the "second-order" expansion of the MIS model cannot be considered complete. Even though the dissipative terms are based on kinetic theory, the model contains only squares of firstorder "thermodynamic fluxes" (as in the sense of Onsager [10]) in all possible combinations (see Andersson and Comer [11], Haskell et al. [12] for Newtonian multi-fluid applications). Last, but not least, the equations of motion are obtained from the conservation of the total stress-energy-momentum tensor of the system, and it is not clear how to extend the model to multi-fluid systems relevant in, say, astrophysics and cosmology.

Another important step, at least from the formal point of view, was taken by Carter [13]. His model is based on a variational principle in which thermodynamic fluxes are upgraded to dynamical variables. However, in order to complete the identification of these new dynamical fields with the usual thermodynamic fluxes, a specific expansion in deviation from thermal equilibrium had to be introduced, and the resulting model was shown to belong to the same 
family as those of the MIS variety (see [14]).

More recently, a fairly general procedure for deriving the field equations for dissipative fluids from an action principle has been put forward by Andersson and Comer [15]. It extends the convective variational principle for perfect multi-fluids introduced by Carter [16] to include dissipation - notably, maintaining the particle fluxes as the only dynamical fields. Moreover, the action and the field equations it produces are fully non-linear. The "variational" aspect of the approach is in the context of the action principle, and even though it may sound counter-intuitive, there is nothing in the variational process that says the field equations themselves have to be linear in the fields.

An important - more familiar - example illustrating this same feature is the variational principle for the Einstein-Hilbert action for General Relativity. It yields the Einstein Equations, which are notoriously non-linear in the metric. Another useful example is the action for Quantum Chromodynamics, which uses non-Abelian gauge-vector bosons. The key point is that, unlike the MIS approach, the Andersson and Comer [15] action principle does not reference any sort of chemical, dynamical, or thermal equilibrium, other than to start with the assumption that the physics can be modelled as fluid phenomena.

The main goal of the present work is to compare the action-based formulation with previous approaches (such as MIS). They-and more recent works [17, 18] —use an expansion to create an approximate set of field equations to describe dissipative phenomena. Since the action-based model already provides a set of equations (at least in principle) valid in every regime, we can make the comparison using standard perturbation techniques. The dissipation terms are assumed to generate first-order deviations away from equilibria obtained using the non-dissipative limit of the field equations. Working this way we hope to also understand better the role of length- and time-scales of fluid elements on the large scale behavior of the system; in particular, how to link the micro-scale dynamics of the many particles in a fluid element with the macro-scale dynamics between the fluid elements themselves, and the role of the Equivalence Principle in setting these scales.

The paper is laid out as follows: In section II we briefly summarize the action-based model. In section III we discuss the role of equilibrium from the fluid perspective and study the dissipative action-based equations in this limit. In section IV we set the stage for the perturbative expansion around equilibrium, building it directly on the matter space. We also introduce a conceptual novelty, the "equilibrium observer" frame of reference, to be 
distinguished from the Landau or Eckart frames, and show how it arises naturally (as a diffeomorphism) in the formalism. In section $\mathrm{V}$ we impose the (thermodynamically motivated) condition that energy (density) is minimized at equilibrium and show that the dissipative pieces of the field equations vanish in this limit. This is a novel result because it is normally assumed. In sections VI and VII we complete the expansion and identify the thermodynamic fluxes in the variational context. In section VII B we provide an explicit example by applying the formalism to a single viscous fluid, which also provides context for discussing the key issue of causality. Finally, in section VIII, we sketch out how to implement a causal response, even at first order in the expansion, by using equations of the telegraph type for the fluxes.

Before moving on it is useful to clarify the index notation used throughout the discussion. We use latin letters $a, b, c, \ldots$ for spacetime indices, and roman letters-such as $\mathrm{x}, \mathrm{y}$ (or $\mathrm{n}, \mathrm{s}$ in specific models) - to label different chemical species and to distinguish dissipative (d) from equilibrium (e) processes. Capital letters $A, B, C, \ldots$ are used for matter-space indices (see below). Note that the Einstein summation convention does not apply to the chemical indices.

\section{ACTION-BASED APPROACH TO DISSIPATIVE FLUIDS: A BRIEF RECAP}

\section{A. Flux Definition}

The crux of the fluid modelling scheme is to assume that knowledge of the total massenergy and momentum flux obtained by tracking the worldlines of individual particles can be replaced with tracking the worldlines of fluid elements, which are defined in the following

way: Take a multi-particle system at some initial time having, say, total spatial size $V$, total number of particles $N$, total mass-energy $E$, and total entropy $S$. At the same time, fill-up side-to-side, top-to-bottom, and front-to-back the entire system with $I=1 \ldots M$ local conceptual boxes - the fluid elements. Each element has its own volume $\delta V_{I}$, number of particles $\delta N_{I}$, mass-energy $\delta E_{I}$, and entropy $\delta S_{I}$. Roughly speaking, if there are characteristic values $\delta V_{I} \sim \delta V, \delta N_{I} \sim \delta N$, etcetera, representative of the fluid elements, then $V \sim M \delta V, N \sim M \delta N, E \sim M \delta E$, and $S \sim M \delta S$. Clearly, as the number $M$ is increased the ratios $\delta V / V, \delta N / N$, etcetera decrease, and the elements become ultra-local, implying 
that the change in the spacetime metric across them is small.

Now consider the $I^{\text {th }}$-fluid element. It moves through spacetime and, if the element is small enough, the trajectory can be accurately represented by a single unit four-velocity $u_{I}^{a}$. When taken together, and in the limit $M \rightarrow \infty$, all the $u_{I}^{a}$ form a vector field on spacetime and this field plays a role in the fluid system's degrees of freedom. If a local typical scattering length $\lambda_{I}$ between the particles exists, and the size of fluid elements is commensurate with that length $\left(\delta V_{I} \sim \lambda_{I}^{3}\right)$, then the average four-velocity of the $\delta N_{I}$ particles will be $u_{I}^{a}$.

In principle, we now have everything we need to define the actual fluid degrees of freedom, which are the particle fluxes $n_{I}^{a}=\left(\delta N_{I} / \delta V_{I}\right) u_{I}^{a}$. But, the fact that we have introduced typical scattering lengths and average velocities as part of our fluid element definition means we have assumed that fluid elements contain enough particles to warrant a statistical/thermodynamical treatment; i.e., we have to know how the individual four-momenta of the particles are distributed initially with respect to the fluid elements, and then redistributed as the fluid evolves. It is this redistribution process that leads to dissipation and is so difficult to model.

\section{B. The Action Principle}

Now we will outline the action-based approach to dissipation. First, recall that an action is a functional of the fundamental fields of the system under study (here, the particle flux currents and the spacetime metric). It is a measure, of sorts, on the set of all possible field configurations. Roughly, the total set can be separated into two subsets: 1) those which are completely arbitrary in the sense that they are not solutions of field equations; and, 2) those which are not arbitrary and represent solutions of field equations. It is well-established that the second subset of configurations are those which lead to extrema of the action; that is, take arbitrary field configurations from subset 1) which are close to configurations from subset 2), expand the action to first-order in their difference, and then determine if generic restrictions on the field configurations exist which makes the first-order difference term in the expansion vanish. If the action principle is well-formulated, then generic restrictions will exist and will self-consistently be the field equations which define the field configuration subset 2) above. There is nothing in this process that restricts the field equations to be 
linear in subset 2) configurations. ${ }^{1}$

Denoting the four-current particle fluxes of the various species $^{2}$ as $n_{\mathrm{x}}^{a}$ (with $\mathrm{x}$ a label identifying the species), the functional dependence of the Lagrangian density becomes $\sqrt{|g|} \Lambda\left(n_{\mathrm{x}}^{2}, n_{\mathrm{xy}}^{2}\right)$ where

$$
\begin{gathered}
n_{\mathrm{x}}^{2}=-n_{\mathrm{x}}^{a} n_{\mathrm{x}}^{b} g_{a b}, \\
n_{\mathrm{xy}}^{2}=-n_{\mathrm{x}}^{a} n_{\mathrm{y}}^{b} g_{a b} .
\end{gathered}
$$

A nice feature of the variational approach is that it naturally provides the conjugate fourmomenta of each species while accounting for the entrainment effect (see, for example, [19, 20]). Roughly speaking, entrainment causes a species' four-momentum $\mu_{a}^{\mathrm{x}}$ to be misaligned with its respective particle flux $n_{\mathrm{x}}^{a}$. We see this explicitly in the momentum/flux relation which is given by

$$
\mu_{a}^{\mathrm{x}}=\mathcal{B}^{\mathrm{x}} n_{a}^{\mathrm{x}}+\sum_{\mathrm{y} \neq \mathrm{x}} \mathcal{A}^{\mathrm{xy}} n_{a}^{\mathrm{y}}
$$

where

$$
\mathcal{B}^{\mathrm{x}}=-2 \frac{\partial \Lambda}{\partial n_{\mathrm{x}}^{2}}
$$

while the entrainment coefficients are defined as

$$
\mathcal{A}^{\mathrm{xy}}=-\frac{\partial \Lambda}{\partial n_{\mathrm{xy}}^{2}} .
$$

It is well established that, to obtain non-trivial fluid equations of motion from such a Lagrangian, the variation of the particle fluxes must be constrained [21, 22].

\section{The Matter Space Formulation and Field Equations}

A particularly elegant way of imposing the relevant constraint involves introducing the matter space, defined by identifying each current worldline as a single point, see [23]. For

${ }^{1}$ For example, a scalar field theory with $V(\phi)=\lambda \phi^{4}$ potential will yield field equations which are non-linear in $\phi$.

${ }^{2}$ Hereafter we will not make any distinction between the words species and constituents. For instance, in a neutron star context, the protons and the electrons constitute examples of chemical species/constituents. When considered individually, it would be natural to think of the particle species, but when the two are locked together (leading to a charge-neutral conglomerate) the word constituent may be more appropriate. However, we do not need to make a distinction for the present discussion. 
each fluid, the matter space is a three-dimensional manifold, so that when we introduce a set of coordinates $X_{\mathrm{x}}^{A}$ on, say, the x-fluid's matter space, we give a "name", or label, to each fluid element. Because the entire worldline of each fluid element is mapped to a single matter space point, it is clear that the fluid element's label $X_{\mathrm{x}}^{A}$, now considered as a collection of three scalars on spacetime, takes the same value at each point on the worldline. After assigning a label to each fluid element worldline, we can use the linear map

$$
\Psi_{\mathrm{x} a}^{A} \doteq \frac{\partial X_{\mathrm{x}}^{A}}{\partial x^{a}}
$$

to push-forward (pull-back) vectors (co-vectors) between spacetime and the matter spaces. This is important because we can associate with each of the particle fluxes $n_{\mathrm{x}}^{a}$ a three-form $n_{a b c}^{\mathrm{x}}$ by the standard Hodge-dual procedure ${ }^{3}$ :

$$
n_{\mathrm{x}}^{a}=\frac{1}{3 !} \varepsilon^{b c d a} n_{b c d}^{\mathrm{x}}, \quad n_{a b c}^{\mathrm{x}}=\varepsilon_{e a b c} n_{\mathrm{x}}^{e}
$$

Now we can assume that the spacetime three-form $n_{a b c}^{\mathrm{x}}$ is obtained by pulling back a corresponding matter space three-form, to be denoted $n_{A B C}^{\mathrm{x}}$; namely,

$$
n_{a b c}^{\mathrm{x}}=\Psi_{\mathrm{x}[a}^{A} \Psi_{\mathrm{x} b}^{B} \Psi_{\mathrm{x} c]}^{C} n_{A B C}^{\mathrm{x}}
$$

where, as usual, straight brackets indicate anti-symmetrization (and round ones symmetrization). Similarly, upon applying the Hodge-dual process to the four-momentum $\mu_{a}^{\mathrm{x}}$, we can push-forward with the map and identify a matter space momentum "three-form" $\mu_{\mathrm{x}}^{A B C}$ via

$$
\begin{aligned}
\mu_{\mathrm{x}}^{a b c} & =\varepsilon^{d a b c} \mu_{d}^{\mathrm{x}} \\
\mu_{\mathrm{x}}^{A B C} & =\Psi_{\mathrm{x}[a}^{A} \Psi_{\mathrm{x} b}^{B} \Psi_{\mathrm{x} c]}^{C} \mu_{\mathrm{x}}^{a b c} .
\end{aligned}
$$

The main idea of the convective variational principle is to obtain the particle flux variation $\delta n_{\mathrm{x}}^{a}$ by first varying the matter-space three-form and then working backwards.

Generally speaking, there are two ways of tracking changes in a fluid system-Eulerian and Lagrangian. The first, to be denoted by a $\delta$, measures changes in the fluid with respect to frames defined by the spacetime coordinates. The second, to be denoted $\Delta_{\mathrm{x}}$, measures

\footnotetext{
${ }^{3}$ Here, we follow the Hodge-dual convention of [24] and have used $\varepsilon^{b c d a} \varepsilon_{e b c d}=-3 ! \delta_{e}^{a}$ to establish a sign convention for the dual $\varepsilon^{b c d a}$ of the spacetime measure form.
} 
changes with respect to fluid elements. Locally, the two can be related through the Lie derivative along some displacement vector field. If $\xi_{\mathrm{x}}^{a}$ is the displacement field, then ${ }^{4}$

$$
\Delta_{\mathrm{x}}=\delta+\mathcal{L}_{\xi_{\mathrm{x}}}
$$

where $\mathcal{L}_{\xi_{\mathrm{x}}}$ is the Lie derivative with respect to $\xi_{\mathrm{x}}^{a}$. Because the label $X_{\mathrm{x}}^{A}$ of a fluid element is fixed, we can assert

$$
\Delta X_{\mathrm{x}}^{A}=0 \Longrightarrow \delta X_{\mathrm{x}}^{A}=-\mathcal{L}_{\xi_{\mathrm{x}}} X_{\mathrm{x}}^{A}=-\Psi_{\mathrm{x} a}^{A} \xi_{\mathrm{x}}^{a},
$$

and thereby lock any displacement vector $\xi_{\mathrm{x}}^{a}$ on spacetime to a displacement $\delta X_{\mathrm{x}}^{A}$ on matter space. Now, it is easy to show that the particle flux variation $\delta n_{\mathrm{x}}^{a}$ is (see [15])

$$
\delta n_{\mathrm{x}}^{a}=-\frac{1}{2} n_{\mathrm{x}}^{a} g^{b c} \delta g_{b c}-\frac{1}{3 !} \varepsilon^{b c d a}\left(\mathcal{L}_{\xi_{\mathrm{x}}} n_{b c d}^{\mathrm{x}}-\Psi_{\mathrm{x}[b}^{B} \Psi_{\mathrm{x} c}^{C} \Psi_{\mathrm{x} d]}^{D} \Delta_{\mathrm{x}} n_{B C D}^{\mathrm{x}}\right),
$$

Formally, we can take $n_{A B C}^{\mathrm{x}}$ to be a particle measure form on the matter space, which "counts" the total number of species x particles in the system. If it is a tensor on matter space then it must be a function only of the matter space coordinates $X_{\mathrm{x}}^{A}$. But recall that in spacetime, the matter space labels are scalar fields $X_{\mathrm{x}}^{A}\left(x^{a}\right)$ with the special property that they take the same value at each spacetime point on the fluid element worldline with which they are associated; clearly, this means that $n_{A B C}^{\mathrm{x}}$ also takes the same value with respect to its particular fluid element worldline. The net impact is that $n_{a b c}^{\mathrm{x}}$ is automatically closed-because $n_{A B C}^{\mathrm{x}}$ is a three-form on a 3-dimensional matter space - and therefore the particle flux is conserved ${ }^{5}$ :

$$
\nabla_{a} n_{\mathrm{x}}^{a}=\frac{1}{3 !} \varepsilon^{b c d a} \nabla_{[a} n_{b c d]}^{\mathrm{x}}=\frac{1}{4 !} \varepsilon^{b c d a}(d n)_{a b c d}=0 .
$$

But there is a deeper point to be made here.

The fact that $n_{A B C}^{\mathrm{x}}=n_{A B C}^{\mathrm{x}}\left(X_{\mathrm{x}}^{A}\right)$ implies $\Delta_{\mathrm{x}} n_{A B C}^{\mathrm{x}}=0$, and one can verify that the flux variation above reduces to the well-known result for non-dissipative fluids. Therefore, to get the non-dissipative equations of motion one simply has to impose that the number of particles is conserved in the variation, or, equivalently, that the particle creation rates

\footnotetext{
${ }^{4}$ We note that this relation between Lagrangian and Eulerian variation works to first order in the perturbation fields $\xi_{\mathrm{x}}^{a}$, see Friedman and Schutz [25] for further details.

${ }^{5}$ Here with $d n$ we mean the exterior derivative of the differential form $n$.
} 
$\Gamma_{\mathrm{x}}=\nabla_{a} n_{\mathrm{x}}^{a}$ vanish. It then follows [15] that a way to include dissipative processes (read: $\left.\Gamma_{\mathrm{x}} \neq 0\right)$ at the level of the action principle is to break the matter space tensorial nature of the particle measure form $n_{A B C}^{\mathrm{x}}$ and allow it to be a function of more than just the $X_{\mathrm{x}}^{A}$; in other words, we break the closure property of the $n_{a b c}^{\mathrm{x}}$.

This general strategy has been applied by Andersson and Comer [15], who focused on the cases where $n_{A B C}^{\mathrm{x}}$ depends on other species matter space coordinates $X_{\mathrm{y}}^{A}$ as well as the projected metrics

$$
\begin{aligned}
& g_{\mathrm{x}}^{A B}=\Psi_{\mathrm{x} a}^{A} \Psi_{\mathrm{x} b}^{B} g^{a b}, \\
& g_{\mathrm{y}}^{A B}=\Psi_{\mathrm{y} a}^{A} \Psi_{\mathrm{y} b}^{B} g^{a b}, \\
& g_{\mathrm{xy}}^{A B}=\Psi_{\mathrm{x} a}^{A} \Psi_{\mathrm{y} b}^{B} g^{a b} .
\end{aligned}
$$

This additional functional dependence in the particle measure forms $n_{A B C}^{\mathrm{x}}$ turns out to produce additional terms in the equations of motion representing different dissipation channels. Specifically, the equations of motion take the form

$$
f_{a}^{\mathrm{x}}+\Gamma_{\mathrm{x}} \mu_{a}^{\mathrm{x}}=-\nabla^{b} D_{b a}^{\mathrm{x}}+R_{a}^{\mathrm{x}},
$$

where

$$
\begin{aligned}
f_{a}^{\mathrm{x}} & =2 n_{\mathrm{x}}^{b} \nabla_{[b} \mu_{a]}^{\mathrm{x}}, \\
D_{a b}^{\mathrm{x}} & =S_{a b}^{\mathrm{x}}+\sum_{\mathrm{y} \neq \mathrm{x}} s_{a b}^{\mathrm{yx}}+\frac{1}{2}\left(\mathcal{S}_{b a}^{\mathrm{xy}}+\mathcal{S}_{a b}^{\mathrm{yx}}\right), \\
R_{a}^{\mathrm{x}} & =\sum_{\mathrm{y} \neq \mathrm{x}}\left(\mathrm{R}_{a}^{\mathrm{yx}}-\mathrm{R}_{a}^{\mathrm{xy}}\right)+\left(r_{a}^{\mathrm{yx}}-r_{a}^{\mathrm{xy}}\right)+\left(\mathcal{R}_{a}^{\mathrm{yx}}-\mathcal{R}_{a}^{\mathrm{xy}}\right) .
\end{aligned}
$$

Projecting the field equation along $u_{\mathrm{x}}^{a}=n_{\mathrm{x}}^{a} / n_{\mathrm{x}}$, we see that

$$
\left(-u_{\mathrm{x}}^{a} \mu_{a}^{\mathrm{x}}\right) \Gamma_{\mathrm{x}}=u_{\mathrm{x}}^{a} \nabla^{b} D_{b a}^{\mathrm{x}}-u_{\mathrm{x}}^{a} R_{a}^{\mathrm{x}},
$$

while the stress-energy-momentum tensor is

$$
T_{a b}=\Psi g_{a b}+\sum_{\mathrm{x}}\left(n_{a}^{\mathrm{x}} \mu_{b}^{\mathrm{x}}+D_{a b}^{\mathrm{x}}\right) .
$$

It follows, as an identity, that $\sum_{\mathrm{x}} R_{a}^{\mathrm{x}}=0$, and because of this we have automatically $\nabla^{a} T_{a b}=0$. Finally, it is the case that $u_{\mathrm{x}}^{b} D_{a b}^{\mathrm{x}}=0$ automatically.

The explicit expressions for the $\mathcal{R}_{a}^{\mathrm{yx}}, S_{a b}^{\mathrm{x}}$, etcetera terms will be given later, at a point where their application is more relevant. However, it is important to note here that the 
"resistive terms" $r_{a}^{\mathrm{xy}}, \mathcal{R}_{a}^{\mathrm{xy}}$ as well as the viscous tensors $s_{a b}^{\mathrm{xy}}, \mathcal{S}_{a b}^{\mathrm{xy}}$ arise because we assume that $n_{A B C}^{\mathrm{x}}$ depends on $g_{\mathrm{y}}^{A B}$ and $g_{\mathrm{xy}}^{A B}$, respectively. Also, it is easy to see that in general the x-species total viscous tensor $D_{a b}^{\mathrm{x}}$ is not necessarily symmetric because $\mathcal{S}_{a b}^{\mathrm{xy}}$ is not. This property is, however, not inherited by the total viscous tensor of the system meaning $D_{a b}=$ $\sum_{\mathrm{x}} D_{a b}^{\mathrm{x}}=D_{b a}$.

\section{Matter Space Volume Forms}

All dissipative terms that enter the action-based equations are obtained by assuming that the fundamental current three-forms $n_{a b c}^{\mathrm{x}}$ depend on an additional set of quantities which breaks their closure $\left(\nabla_{[a} n_{b c d]}^{\mathrm{x}} \neq 0\right)$. We now want to explain how this can happen, but begin by introducing a bit of notation.

We need to distinguish between the Levi-Civita symbol $\eta_{A B C}$ and a volume measure form $\varepsilon_{A B C}^{\mathrm{x}}$ on the matter space. The Levi-Civita symbol is defined as $\eta_{A B C}=[A B C]$ for every chosen set of coordinates (and thus is not a tensor but a tensor density) while the volume measure form $\varepsilon_{A B C}^{\mathrm{x}}$ can be defined ${ }^{6}$ by means of the push-forward of the metric:

$$
\begin{aligned}
& g_{\mathrm{x}}=\frac{1}{3 !} \eta_{A B C} \eta_{D E F} g_{\mathrm{x}}^{A D} g_{\mathrm{x}}^{B E} g_{\mathrm{x}}^{C F}=\operatorname{det}\left(g_{\mathrm{x}}^{A B}\right) \\
& \varepsilon_{A B C}^{\mathrm{x}}=\sqrt{g^{\mathrm{x}}} \eta_{A B C}=\sqrt{g^{\mathrm{x}}}[A B C]
\end{aligned}
$$

where $g^{\mathrm{x}}=\left(g_{\mathrm{x}}\right)^{-1}$ is the determinant of the inverse matrix $g_{A B}^{\mathrm{x}}$; i.e. $g_{A C}^{\mathrm{x}} g_{\mathrm{x}}^{C B}=\delta_{A}^{B}$.

This volume measure form provides a way to measure the volume of "matter elements", infinitesimal volumes in the matter space manifold. We can relate these quantities to the current and momentum three-forms

$$
\begin{aligned}
& n_{A B C}^{\mathrm{x}}=\mathcal{N}_{\mathrm{x}} \varepsilon_{A B C}^{\mathrm{x}}=\overline{\mathcal{N}}_{\mathrm{x}} \eta_{A B C}^{\mathrm{x}}, \\
& \mu_{\mathrm{x}}^{A B C}=\mathcal{M}_{\mathrm{x}} \varepsilon_{\mathrm{x}}^{A B C}=\overline{\mathcal{M}}_{\mathrm{x}} \eta_{\mathrm{x}}^{A B C} .
\end{aligned}
$$

The point we want to make here is that the barred quantities look more like scalar densities on the x-matter space, while the non-barred ones look more like scalars. The relation

\footnotetext{
${ }^{6}$ This is tricky for a couple of reasons: It is well known from work on general relativistic elastic bodies [26] that this is not the only possible choice. Also, the projected metric $g_{\mathrm{x}}^{A B}$ is not "fixed" in the sense that the spacetime metric $g_{a b}$ changes, in a general curved spacetime, as a fluid element moves from point-to-point along its worldline.
} 
between the two normalizations is simply

$$
\begin{gathered}
\mathcal{N}_{\mathrm{x}}=\sqrt{g_{\mathrm{x}}} \overline{\mathcal{N}}_{\mathrm{x}} \\
\mathcal{M}_{\mathrm{x}}=\sqrt{g^{\mathrm{x}}} \overline{\mathcal{M}}_{\mathrm{x}} .
\end{gathered}
$$

We can use this to expedite our use of the convective variational principle by focusing the additional functional dependence of $n_{A B C}^{\mathrm{x}}$ into

$$
\mathcal{N}_{\mathrm{x}}=\mathcal{N}_{\mathrm{x}}\left(X_{\mathrm{x}}^{A}, X_{\mathrm{y}}^{A}, g_{\mathrm{x}}^{A B}, g_{\mathrm{y}}^{A B}, g_{\mathrm{xy}}^{A B}\right)
$$

To make contact with proper quantities measured in spacetime - that is, with the rest frame density and rest frame momentum for each fluid component - it is useful to introduce an appropriate tetrad $e_{a}^{\hat{a}}$ for each species; an orthonormal basis whose timelike unit vector $\boldsymbol{e}_{\hat{0}}=\boldsymbol{u}_{\mathrm{x}}$, so that $u_{\mathrm{x}}^{\hat{a}}=\left(\boldsymbol{e}_{\hat{0}}\right)^{\hat{a}}=\delta_{\hat{0}}^{\hat{a}}=(1,0,0,0)^{T}$. The components of the spacetime measure form in this tetrad basis are ${ }^{7}$

$$
\varepsilon^{\hat{a} \hat{b} \hat{c} \hat{d}}=\varepsilon^{a b c d} e_{a}^{\hat{a}} e_{b}^{\hat{b}} e_{c}^{\hat{c}} e_{d}^{\hat{d}}=\eta^{\hat{a} \hat{b} \hat{c} \hat{d}}
$$

where $\eta^{\hat{a} \hat{b} \hat{c} \hat{d}}=-[\hat{a} \hat{b} \hat{c} \hat{d}]$ and we have omitted the chemical index. Now, since push-forward (and pull-back) is a linear map between vector spaces (the tangent space), it transforms as a linear map under coordinate changes, and we can write

$$
A^{A}=\frac{\partial X_{\mathrm{x}}^{A}}{\partial x^{a}} A^{a}=\Psi_{\mathrm{x} \hat{a}}^{A} A^{\hat{a}}
$$

where we have introduced the short-hand notation ${ }^{8}$

$$
\Psi_{\mathrm{x} \hat{a}}^{A} \equiv \Psi_{\mathrm{x} a}^{A} e_{\hat{a}}^{a}=\frac{\partial X_{\mathrm{x}}^{A}}{\partial x^{a}} e_{\hat{a}}^{a}
$$

Making use of the fact that $0=u_{\mathrm{x}}^{\hat{a}} \Psi_{\mathrm{x} \hat{a}}^{A}=\Psi_{\mathrm{x} \hat{0}}^{A}$ we then get $^{9}$

$$
g_{\mathrm{x}}^{A B}=\Psi_{\mathrm{x} \hat{a}}^{A} \Psi_{\mathrm{x} \hat{b}}^{B} \eta^{\hat{a} \hat{b}} \Longrightarrow g_{\mathrm{x}}=\operatorname{det}\left(\Psi_{\mathrm{x} \hat{i}}^{A}\right)^{2}
$$

which leads to ${ }^{10}$

$$
\begin{aligned}
\mathcal{M}_{\mathrm{x}} & =\frac{1}{3 !} \mu_{\mathrm{x}}^{A B C} \varepsilon_{A B C}^{\mathrm{x}}= \\
& =\frac{1}{3 !} \sqrt{g^{\mathrm{x}}} \eta_{A B C} \Psi_{\mathrm{x} \hat{a}}^{A} \Psi_{\mathrm{x} \hat{b}}^{B} \Psi_{\mathrm{x} \hat{c}}^{C} \varepsilon^{\hat{0} \hat{a} \hat{b} \hat{c}} \mu_{\hat{0}}^{\mathrm{x}}=\mu_{\mathrm{x}}
\end{aligned}
$$

\footnotetext{
${ }^{7}$ Recall that, since $g_{a b}=e_{a}^{\hat{a}} e_{b}^{\hat{b}} \eta_{\hat{a} \hat{b}}$, the determinant of the tetrad $e=\sqrt{|g|}$.

${ }^{8}$ Following [24] we denote the inverse matrix of the tetrad as $e_{\hat{a}}^{a}$.

${ }^{9}$ The index $\hat{i}$ runs over the 1,2,3 components of the tetrad basis, and $\hat{a}=\hat{0}, \hat{i}$.

${ }^{10}$ Note that, because of the standard convention we use $\eta^{\hat{0} \hat{b} \hat{c} \hat{d}}=-\varepsilon^{\hat{b} \hat{c} \hat{d}}$ with $\hat{b}, \hat{c}, \hat{d}=1,2,3$.
} 
where we have used $\mu_{\mathrm{x}}=-\mu_{a}^{\mathrm{x}} u_{\mathrm{x}}^{a}=-\mu_{\mathrm{x}}^{\hat{0}}$. This fact is important because it makes clear that only the (rest-frame) energy content of the four-momentum co-vector $\mu_{a}^{\mathrm{x}}$ is stored in the normalization of the matter space momentum three-form $\mu_{\mathrm{x}}^{A B C}$. Similarly, one can show that $\mathcal{N}_{\mathrm{x}}=n_{\mathrm{x}}$, in fact

$$
\begin{aligned}
n_{\mathrm{x}} & =-\frac{1}{3 !} u_{a}^{\mathrm{x}} \varepsilon^{b c d a} n_{b c d}^{\mathrm{x}}=-\frac{1}{3 !} u_{\hat{a}}^{\mathrm{x}} \varepsilon^{\hat{b} \hat{c} \hat{d} \hat{a}} \Psi_{\mathrm{x}[\hat{b}}^{B} \Psi_{\mathrm{x} \hat{c}}^{C} \Psi_{\mathrm{xd}]}^{D} \varepsilon_{B C D}^{\mathrm{x}} \mathcal{N}_{\mathrm{x}} \\
& =-\frac{1}{3 !} u_{\hat{0}}^{\mathrm{x}} \varepsilon^{\hat{b} \hat{c} \hat{d} \hat{0}} \varepsilon_{\hat{b} \hat{c} \hat{d}} \mathcal{N}_{\mathrm{x}}=-u_{\hat{0}}^{\mathrm{x}} \mathcal{N}_{\mathrm{x}}=\mathcal{N}_{\mathrm{x}} .
\end{aligned}
$$

These relations are not surprising. It is quite intuitive that the non-barred quantities are related to spacetime (rest-frame) densities given that the three-forms $\varepsilon_{A B C}^{\mathrm{x}}$ measure the volume of the matter space elements.

We can also use the tetrad formalism to prove another result that will be needed later on; the intimate connection between a non-zero particle creation rate and an extended functional dependence of the current three-form. In fact, we have (see eq. (12))

$$
\Gamma_{\mathrm{x}}=\nabla_{a} n_{\mathrm{x}}^{a}=\frac{1}{3 !} \varepsilon^{b c d a} \Psi_{\mathrm{x}[b}^{B} \Psi_{\mathrm{x} c}^{C} \Psi_{\mathrm{x} d}^{D} \nabla_{a]} n_{B C D}^{\mathrm{x}}
$$

where we used $\nabla_{[a} \Psi_{\mathrm{x} b}^{B} \Psi_{\mathrm{x} c}^{C} \Psi_{\mathrm{x} d]}^{D}=0$. Introducing (again) a tetrad comoving with the $\mathrm{x}-$ species, and multiplying by $\mu_{\mathrm{x}}$ we have

$$
\mu_{\mathrm{x}} \Gamma_{\mathrm{x}}=\frac{1}{3 !} \mu_{\mathrm{x}}^{A B C} u_{\mathrm{x}}^{a} \nabla_{a} n_{A B C}^{\mathrm{x}} \equiv \frac{1}{3 !} \mu_{\mathrm{x}}^{A B C} \frac{d n_{A B C}^{\mathrm{x}}}{d \tau_{\mathrm{x}}} .
$$

As explained earlier, the right-hand-side of this equation vanishes identically if $n_{A B C}^{\mathrm{x}}=$ $n_{A B C}^{\mathrm{x}}\left(X_{\mathrm{x}}^{A}\right)$, while it is in general non-zero if we assume the extended functional dependence given in eq. (21).

We can now use the introduced normalizations to slim the notation (with respect to that used in [15]) for the various pieces of $R_{a}^{\mathrm{x}}$ and $D_{a b}^{\mathrm{x}}$ which were introduced but not defined above. For instance, the "purely reactive" term from [15] becomes

$$
\mathrm{R}_{a}^{\mathrm{xy}}=\frac{1}{3 !} \mu_{\mathrm{x}}^{A B C} \frac{\partial n_{A B C}^{\mathrm{x}}}{\partial X_{\mathrm{y}}^{D}} \Psi_{\mathrm{y} a}^{D}=\mathcal{M}_{\mathrm{x}} \frac{\partial \mathcal{N}_{\mathrm{x}}}{\partial X_{\mathrm{y}}^{D}} \Psi_{\mathrm{y} a}^{D} \equiv \mathrm{R}_{D}^{\mathrm{xy}} \Psi_{\mathrm{y} a}^{D} .
$$

Similarly we can write

$$
\begin{aligned}
s_{a b}^{\mathrm{xy}} & =\frac{1}{3} \mu_{\mathrm{x}}^{A B C} \frac{\partial n_{A B C}^{\mathrm{x}}}{\partial g_{\mathrm{y}}^{D E}} \Psi_{\mathrm{y} a}^{D} \Psi_{\mathrm{y} b}^{E}=2 \mathcal{M}_{\mathrm{x}} \frac{\partial \mathcal{N}_{\mathrm{x}}}{\partial g_{\mathrm{y}}^{D E}} \Psi_{\mathrm{y} a}^{D} \Psi_{\mathrm{y} b}^{E} \\
& \equiv s_{D E}^{\mathrm{xy}} \Psi_{\mathrm{y} a}^{D} \Psi_{\mathrm{y} b}^{E}, \\
\mathcal{S}_{a b}^{\mathrm{xy}} & =\frac{1}{3} \mu_{\mathrm{x}}^{A B C} \frac{\partial n_{A B C}^{\mathrm{x}}}{\partial g_{\mathrm{xy}}^{D E}} \Psi_{\mathrm{x} a}^{D} \Psi_{\mathrm{y} b}^{E}=2 \mathcal{M}_{\mathrm{x}} \frac{\partial \mathcal{N}_{\mathrm{x}}}{\partial g_{\mathrm{xy}}^{D E}} \Psi_{\mathrm{x} a}^{D} \Psi_{\mathrm{y} b}^{E} \\
& \equiv \mathcal{S}_{D E}^{\mathrm{xy}} \Psi_{\mathrm{x} a}^{D} \Psi_{\mathrm{y} b}^{E},
\end{aligned}
$$


where we have used the fact that the partial derivatives are performed, say, with respect to the metric $g_{\mathrm{y}}^{A B}$ keeping fixed $g_{\mathrm{x}}^{A B}$ and $g_{\mathrm{xy}}^{A B}$. We will consider the validity of this assumption later. The remaining viscous stress tensor, $S_{a b}^{\mathrm{x}}$, leads to a slightly more involved expression, because of the presence of $g^{\mathrm{x}}$ in eq. (20). We have

$$
\begin{aligned}
S_{a b}^{\mathrm{x}} & =\frac{1}{3} \mu_{\mathrm{x}}^{A B C} \frac{\partial n_{A B C}^{\mathrm{x}}}{\partial g_{\mathrm{x}}^{D E}} \Psi_{\mathrm{x} a}^{D} \Psi_{\mathrm{x} b}^{E}=2\left(\frac{\mathcal{M}_{\mathrm{x}}}{\sqrt{g^{\mathrm{x}}}} \frac{\partial\left(\mathcal{N}_{\mathrm{x}} \sqrt{g^{\mathrm{x}}}\right)}{\partial g_{\mathrm{x}}^{D E}}\right) \Psi_{\mathrm{x} a}^{D} \Psi_{\mathrm{x} b}^{E}= \\
& =2\left(\mathcal{M}_{\mathrm{x}} \frac{\partial \mathcal{N}_{\mathrm{x}}}{\partial g_{\mathrm{x}}^{D E}}-\frac{1}{2} \mathcal{N}_{\mathrm{x}} \mathcal{M}_{\mathrm{x}} g_{D E}^{\mathrm{x}}\right) \Psi_{\mathrm{x} a}^{D} \Psi_{\mathrm{x} b}^{E}= \\
& \equiv S_{D E}^{\mathrm{x}} \Psi_{\mathrm{x} a}^{D} \Psi_{\mathrm{x} b}^{E} .
\end{aligned}
$$

It is also obvious, by looking at the respective definitions, that the reactive terms that stem from the fact that $\mathcal{N}_{\mathrm{x}}$ can depend also on $g_{\mathrm{y}}^{A B}$ and $g_{\mathrm{xy}}^{A B}$ can be now written

$$
\begin{aligned}
& r_{a}^{\mathrm{xy}}=\frac{1}{2} s_{D E}^{\mathrm{xy}} \nabla_{a}\left(g^{b c} \Psi_{\mathrm{y} b}^{D} \Psi_{\mathrm{y} c}^{E}\right) \\
& \mathcal{R}_{a}^{\mathrm{xy}}=\frac{1}{2} \mathcal{S}_{D E}^{\mathrm{xy}} g^{b c} \Psi_{\mathrm{x} b}^{D} \nabla_{a}\left(\Psi_{\mathrm{y} c}^{E}\right) .
\end{aligned}
$$

Before moving on, it is advantageous to consider the simplest non-dissipative fluid model which can be derived from the action above - the ordinary perfect fluid, where all particle species and entropy flow together and the total particle numbers and entropy are conserved individually. The calculation is straightforward [27]. All the fluxes have the same fourvelocity, say, $u^{a}$, and so $n_{\mathrm{x}}^{a}=n_{\mathrm{x}} u^{a}$. If each particle number flux is conserved individually, then

$$
\nabla_{a} n_{\mathrm{x}}^{a}=\nabla_{a}\left(n_{\mathrm{x}} u^{a}\right)=u^{a} \nabla_{a} n_{\mathrm{x}}+n_{\mathrm{x}} \nabla_{a} u^{a}=0 \Longrightarrow u^{a} \nabla_{a} \ln n_{\mathrm{x}}=-\nabla_{a} u^{a} .
$$

Obviously, the total particle flux $n^{a}=\sum_{\mathrm{x}} n_{\mathrm{x}}^{a}$ is also conserved and so we can write as well

$$
u^{a} \nabla_{a} \ln n=-\nabla_{a} u^{a}, n=\sum_{\mathrm{x}} n_{\mathrm{x}} .
$$

Therefore, we have

$$
u^{a} \nabla_{a} \ln n_{\mathrm{x}}-u^{a} \nabla_{a} \ln n=0 \Longrightarrow u^{a} \nabla_{a} \frac{n_{\mathrm{x}}}{n}=0 .
$$

The upshot is that each species fraction $n_{\mathrm{x}} / n$ must also be conserved along the flow, and this includes the entropy as well. This implies that only one matter space is required. In the action principle, this means that for each $\mathrm{x}$ we have $\xi_{\mathrm{x}}^{a}=\xi^{a}$, and there is only one Euler equation of the form

$$
\sum_{\mathrm{x}} f_{a}^{\mathrm{x}}=0,
$$


where the $f_{a}^{\mathrm{x}}$ are exactly as defined before.

\section{THE NON-DISSIPATIVE LIMIT}

We will now begin to develop the process for comparing standard relativistic models for dissipative fluids with that provided by the action principle. Standard approaches [37] start with a definition of equilibrium and then build in dissipation via deviations away from this state. The action principle formally does not require any sort of equilibrium, and provides a fully non-linear set of field equations. Obviously, our first task must be to extract from the non-linear equations a notion of equilibrium. This is not straightforward for various reasons, a key one being that an arbitrary spacetime in General Relativity does not have global temporal, spatial, and rotational invariance. As a first step, we will recall features of the typical laboratory set-ups within which the laws of Chemistry, Dynamics, and Thermodynamics were first established.

\section{A. Typical Laboratory Set-up}

A typical laboratory set-up is essentially local in the spacetime sense, implying there is - to a great deal of precision - temporal, spatial, and rotational invariance. Noether symmetries exist, which lead to energy, momentum, and angular momentum conservation. A clean separation between internal and external influences can be made, and these influences themselves can be manipulated. The effect of long-range, non-screenable forces on the system - for example, gravity - can be ignored. Well-defined (theoretical and experimental/observational) notions of total energy and entropy can be realized. Equilibrium can be defined in the broadest sense by saying the system evolves to a state where its total energy is minimized, or, equally, its total entropy is maximized.

Internal interactions are due to, say, chemical reactions, whereas external interactions are those which distort the system's volume or allow particles and heat to enter or leave through the volume's surface. If a system is in chemical equilibrium internally, we can say that the reactions inside it are running forwards and backwards at such a rate that constituent particle number ratios remain fixed in time. If the given system is in chemical equilibrium with another system, then the chemical potentials of the two will be equal. A 
system in dynamical equilibrium just sits there, with no temporal evolution. Any pressure acting on the system's surface will be balanced by an internal pressure of the same value. Finally, we can say that two systems are in thermal equilibrium when there is no heat flow between them, the end result being equality of their respective temperatures. Now, let us return to the problem at hand-equilibrium when General Relativity cannot be neglected.

\section{B. General Relativistic Set-up}

A general relativistic set-up is problematic from the get-go, because one is hard-pressed to find properties of equilibrium like those just discussed which are workable at all timeand length-scales. Broadly speaking, there seems to be no general relativistic rules on how the local thermodynamics of local (intensive) parameters - chemical potential $\mu$, pressure $p$, and temperature $T$ - connects with some notion of global thermodynamics for global (extensive) parameters - such as the total energy E. An unambiguous extrapolation of the standard definitions of chemical, dynamical, and thermal equilibrium given above to General Relativity is not possible, for reasons to be explained below. There is also the well-known difficulty of identifying the total energy of a region in an arbitrary spacetime, since the Equivalence Principle precludes an ultra-local definition of gravitational energy density. ${ }^{11}$

The reason that the laboratory rules for chemical and thermal equilibrium are not viable in General Relativity was established long ago by Tolman and Ehrenfest [28, 29]: In General Relativity, all forms of energy react to gravity. Temperature and chemical potentials represent forms of energy and can undergo red-shift or blue-shift. There is no one temperature for an isolated system, and so saying "system A is in thermal equilibrium with system B if their temperatures are the same" becomes ambiguous; similarly for chemical equilibrium. As for dynamical equilibrium, a standard undergraduate physics calculation shows that pressure increases with depth in water which nevertheless remains at rest. ${ }^{12}$

Even the use of the word "equilibrium" becomes problematic because it tends to imply that a system in thermal and chemical equilibrium is independent of time, because the total

${ }^{11}$ Of course, for asymptotically flat spacetimes, one can define quantities like the Schwarzschild mass. Gravitational wave energy can be defined but only after averaging over wavelengths.

${ }^{12}$ In this context, we can think of it as resulting from the breaking via gravity of spacelike Killing vectors which lead to space-translation invariance. 
entropy and total particle number do not evolve. In General Relativity, a system which is independent of time occurs only for special spacetimes which have a global timelike Killing vector field. Strictly speaking, this immediately puts the non-dissipative fluid models of Cosmology - the Friedman-Lemaitre-Robertson-Walker solutions - out of the discussion, as the universe is expanding, making it time-dependent; maybe worse, it is not even timesymmetric!

This points to another problem of the notion of total energy in General Relativity and arguments based on the standard understanding of energy conservation: In Special Relativity, the curvature is zero and there is a timelike Killing vector field leading directly to a Noether symmetry for the system and total energy conservation. (There are also Killing vector fields representing rotational and spatial invariance, which lead to Noether symmetries resulting in total angular and linear momentum conservation.) In an expanding universe this line of reasoning for energy conservation obviously breaks down.

The main message is this: Important issues remain unsettled even after a century's worth of debate. We will not resolve these issues here; instead, what we will do is take the action-based formalism and see how its internal machinery can be manipulated to produce a self-consistent notion of the non-dissipative limit, without trying to resolve the deeper issues about the nature of equilibrium. ${ }^{13}$ Our way forward is to take advantage of the fact that the action-based field equations are fully non-linear and complete.

\section{Multiple Equilibrium States}

The main mechanism for manipulating the machinery of the action-based field equations is to apply perturbation techniques similar to those used to determine, say, quasi-normal modes of neutron stars. The general idea for neutron stars is to analyze linear perturbations of configurations having particular symmetries generated by Killing vectors. Among the most studied neutron star "ground-states" are those having Killing vectors which generate staticity and spherical symmetry, and those with Killing vectors that generate axisymmetry and stationarity; basically, non-rotating and rotating backgrounds, respectively.

In an analogous way, we can expect different options for generating the non-dissipative limit of a multi-fluid system. For example, we can take the limit where the different dissipa-

${ }^{13}$ We will still use the word "equilibrium" interchangeably with the non-dissipative limit. 
tion coefficients (such as shear and bulk viscosities) are effectively zero. Another possibility is the limit where the dissipation coefficients are non-zero but the fluid motion itself is such that the dissipation mechanisms are not acting. The formalism developed by Onsager [10] is worthy of mention here, because the system of field equations it creates are more explicit in how the two limits can be implemented (see, for example, [11]). It is interesting also to note that the philosophy of the Onsager approach is not so much about how to expand away from an equilibrium, but rather how a non-equilibrium system gets driven back to the equilibrium state. Here, because the field equations are fully non-linear, they can, in principle, describe systems which are being driven toward or away from equilibrium.

Next, we will explore some of the different options for equilibrium states. We will use a global analysis which assumes that the Second Law of Thermodynamics applies and that a knowledge of the fluxes throughout a region of spacetime is enough to determine whether or not dissipation is acting. A local analysis of the formalism will also be pursued, involving the field equations themselves.

\section{Global Analysis of the Non-dissipative Limit}

Recall that the fundamental dynamical variables are the particle fluxes $n_{\mathrm{x}}^{a}$ and the entropy $s^{a}=n_{\mathrm{s}}^{a}{ }^{14}$ The formalism's linchpin is the breaking of the closure of the particle-flux threeforms, $n_{a b c}^{\mathrm{x}}$ and $s_{a b c}$, which leads to non-zero creation rates $\Gamma_{\mathrm{x}}$ and $\Gamma_{\mathrm{s}}$. In turn, these non-zero creation rates lead to the resistive contribution $R_{\mathrm{x}}^{a}$ and the dissipation tensor $D_{a b}^{\mathrm{x}}$ terms in the equations of motion. The nice thing about fluxes, which we will exploit here, is that they can be integrated.

When we use the Einstein equations and the field equations of a multi-fluid system, our goal is to get solutions for the metric and fluxes on a "chunk" of spacetime, for a given set of initial/boundary conditions. Suppose we pick an ad hoc region $\mathcal{M}$ of spacetime, as illustrated in fig. 1. The fact that it is a region implies there is a "conceptual boundary", meaning the whole spacetime is being divided up into smaller domains. Let $u_{\mathrm{B}}^{a}$ (collectively) denote the unit normal to the total boundary of the region, defined so that it always points "out".

\footnotetext{
${ }^{14}$ Because we impose the Second Law of Thermodynamics below, we are specifically separating out the entropy flux in this discussion.
} 


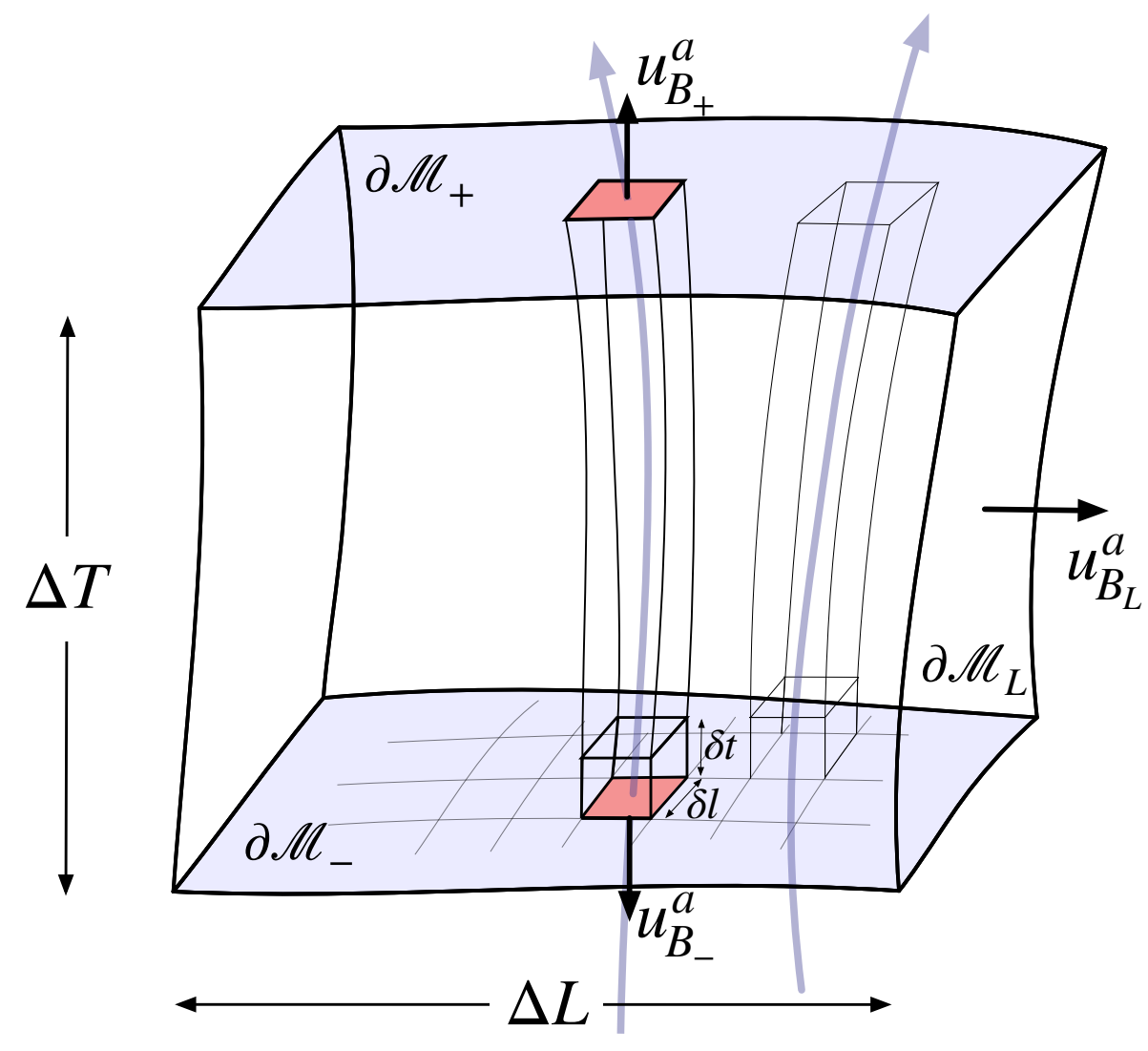

Fig. 1. A depiction of the spacetime region $\mathcal{M}$, with one spatial axis suppressed. It has a characteristic spatial size $\Delta L$ and temporal size $\Delta T$. Inside $\mathcal{M}$ is a smaller region $\delta \mathcal{M}$ of characteristic spatial and temporal size $\delta l$ and $\delta t$, respectively. The boundary $\partial \mathcal{M}$ consists of the initial and final time-slices $\partial \mathcal{M}_{-}, \partial \mathcal{M}_{+}$and the timelike hypersurface $\partial \mathcal{M}_{L}$.

The boundary itself consists of two spacelike hypersurfaces $\partial \mathcal{M}_{ \pm}$(with unit normals $u_{\mathrm{B}_{ \pm}}^{a}$, $u_{\mathrm{B}_{ \pm}}^{a} u_{a}^{\mathrm{B}_{ \pm}}=-1$ ), and a timelike hypersurface $\partial \mathcal{M}_{L}$ (with unit normal $u_{\mathrm{B}_{\mathrm{L}}}^{a}, u_{\mathrm{B}_{\mathrm{L}}}^{a} u_{a}^{\mathrm{B}_{\mathrm{L}}}=+1$ ); in essence, think of $\partial \mathcal{M}_{-}$as a $3 \mathrm{D}$ region of characteristic volume $\Delta L^{3}$ on an initial time-slice of $\mathcal{M}$ and $\partial \mathcal{M}_{+}$as the same volume on the final time-slice, and then $\partial \mathcal{M}_{L}$ will be similar to the union of the surface of the same volume on each leaf of some spacelike foliation of $\mathcal{M}$ between $\partial \mathcal{M}_{-}$and $\partial \mathcal{M}_{+}$. The induced metric on $\partial \mathcal{M}_{ \pm}$is $h_{ \pm}^{a b}=g^{a b}+u_{\mathrm{B}_{ \pm}}^{a} u_{\mathrm{B}_{ \pm}}^{b}$ and for $\partial \mathcal{M}_{L}$ it is $h_{L}^{a b}=g^{a b}-u_{\mathrm{B}_{\mathrm{L}}}^{a} u_{\mathrm{B}_{\mathrm{L}}}^{b}$.

There are three contributions to the total particle number change $\Delta N^{\mathrm{x}}$ and total entropy change $\Delta S$ : (i) The total particle number $N_{-}^{\mathrm{x}}$ and entropy $S_{-}^{\mathrm{x}}$ which exist in $\partial \mathcal{M}_{-}$; (ii) The total particle number $N_{+}^{\mathrm{x}}$ and entropy $S_{+}^{\mathrm{x}}$ which exist in $\partial \mathcal{M}_{+}$; and, (iii) The number of 
particles $\Delta N_{L}^{\mathrm{x}}$ and amount of entropy $\Delta S_{L}$ which enter/leave $\partial \mathcal{M}_{L}$. Each contribution is obtainable from its associated flux: If $n_{ \pm}^{\mathrm{x}}\left(s_{ \pm}\right)$are the particle number (entropy) densities as measured with respect to the volumes $\partial \mathcal{M}_{ \pm}$, and $n_{L}^{\mathrm{x}}\left(s_{L}\right)$ is the number of particles (amount of entropy) per unit area per unit time entering/leaving $\partial \mathcal{M}_{L}$, then

$$
\begin{aligned}
N_{+}^{\mathrm{x}} & =\int_{\partial \mathcal{M}_{+}} \mathrm{d}^{3} x \sqrt{h_{+}} n_{+}^{\mathrm{x}}=\int_{\partial \mathcal{M}_{+}} \mathrm{d}^{3} x \sqrt{h_{+}}\left(-u_{a}^{\mathrm{B}_{+}} n_{\mathrm{x}}^{a}\right), \\
N_{-}^{\mathrm{x}} & =\int_{\partial \mathcal{M}_{-}} \mathrm{d}^{3} x \sqrt{h_{-}} n_{-}^{\mathrm{x}}=\int_{\partial \mathcal{M}_{-}} \mathrm{d}^{3} x \sqrt{h_{-}}\left(u_{a}^{\mathrm{B}} n_{\mathrm{x}}^{a}\right), \\
\Delta N_{L}^{\mathrm{x}} & =\int_{\partial \mathcal{M}_{L}} \mathrm{~d}^{3} x \sqrt{-h_{L}} n_{L}^{\mathrm{x}}=\int_{\partial \mathcal{M}_{L}} \mathrm{~d}^{3} x \sqrt{-h_{L}}\left(u_{a}^{\mathrm{B}_{\mathrm{L}}} n_{\mathrm{x}}^{a}\right)
\end{aligned}
$$

and

$$
\begin{aligned}
S_{+} & =\int_{\partial \mathcal{M}_{+}} \mathrm{d}^{3} x \sqrt{h_{+}} s_{+}=\int_{\partial \mathcal{M}_{+}} \mathrm{d}^{3} x \sqrt{h_{+}}\left(-u_{a}^{\mathrm{B}_{+}} s^{a}\right), \\
S_{-} & =\int_{\partial \mathcal{M}_{-}} \mathrm{d}^{3} x \sqrt{h_{-}} s_{-}=\int_{\partial \mathcal{M}_{-}} \mathrm{d}^{3} x \sqrt{h_{-}}\left(u_{a}^{\mathrm{B}_{-}} s^{a}\right), \\
\Delta S_{L} & =\int_{\partial \mathcal{M}_{L}} \mathrm{~d}^{3} x \sqrt{-h_{L}} s_{L}=\int_{\partial \mathcal{M}_{L}} \mathrm{~d}^{3} x \sqrt{-h_{L}}\left(u_{a}^{\mathrm{B}_{\mathrm{L}}} s^{a}\right) .
\end{aligned}
$$

where we have taken into account the fact that $u_{\mathrm{B}_{-}}^{a}$ points to the past. The changes in the total x-particles $\Delta N_{\mathrm{x}}$ and entropy $\Delta S$ over the region $\mathcal{M}$ are therefore

$$
\begin{array}{r}
\Delta N^{\mathrm{x}}=N_{+}^{\mathrm{x}}-N_{-}^{\mathrm{x}}+\Delta N_{L}^{\mathrm{x}}, \\
\Delta S=S_{+}-S_{-}+\Delta S_{L} .
\end{array}
$$

If the length- and time-scales of spacetime region $\mathcal{M}$ are those typical of terrestrial labs (read: its curvature is zero throughout), then we have great confidence in asserting the Second Law of Thermodynamics; namely, the net change of the total entropy must satisfy $\Delta S \geq 0$. We could even be confident that we could determine the total energy $E$ and volume $V$ of the system, and have a working First Law of Thermodynamics which connects $\Delta E$, $\Delta N^{\mathrm{x}}, \Delta V$, and $\Delta S$ :

$$
\Delta E=T \Delta S-p \Delta V+\sum_{\mathrm{x}} \mu_{\mathrm{x}} \Delta N^{\mathrm{x}}
$$

The temperature $T$, pressure $p$, and chemical potentials $\mu_{\mathrm{x}}$ would be well-defined and calculable. We could even use the standard notions of chemical, dynamical, and thermal equilibrium and say that system A of spacetime region $\mathcal{M}_{A}$ is in chemical, dynamical, and thermal equilibrium with system $\mathrm{B}$ of spacetime region $\mathcal{M}_{B}$ if, respectively, their chemical potentials are equal, their pressures are equal, and their temperatures are equal. 
Now, let us suppose we have a region large enough that spacetime curvature can no longer be ignored. Probably, it would be a safe bet to say that the Second Law still applies; i.e., $\Delta S \geq 0$. But, we are hard-pressed to employ the laboratory definitions of chemical, dynamical, and thermal equilibrium. Consequently, it is difficult to imagine a global First Law of Thermodynamics for general relativistic multifluid systems similar to that in eq. (41); again, the reason being that intensive parameters are spacetime dependent, and an extensive parameter like total energy may not even be definable. Still, our task is to explore any possible link between parameters which require scales where spacetime curvature is necessary $\left(\Delta N^{\mathrm{x}}\right.$ and $\left.\Delta S\right)$ to the local fluid variables $\left(n_{\mathrm{x}}^{a}\right.$ and $\left.s^{a}\right)$ which enter the fluid field equations. Fortunately, the divergence theorem provides such a link.

Applying it to the divergence of both the particle and entropy fluxes gives ${ }^{15}$

$$
\begin{aligned}
\int_{\mathcal{M}} \mathrm{d}^{4} x \sqrt{-g} \nabla_{a} n_{\mathrm{x}}^{a}=-\int_{\partial \mathcal{M}_{+}} \mathrm{d}^{3} x \sqrt{h_{+}}\left(u_{a}^{\mathrm{B}_{+}} n_{\mathrm{x}}^{a}\right) & -\int_{\partial \mathcal{M}_{-}} \mathrm{d}^{3} x \sqrt{h_{-}}\left(u_{a}^{\mathrm{B}_{-}} n_{\mathrm{x}}^{a}\right) \\
& +\int_{\partial \mathcal{M}_{L}} \mathrm{~d}^{3} x \sqrt{-h_{L}}\left(u_{a}^{\mathrm{B}_{\mathrm{L}}} n_{\mathrm{x}}^{a}\right)
\end{aligned}
$$

and

$$
\begin{array}{r}
\int_{\mathcal{M}} \mathrm{d}^{4} x \sqrt{-g} \nabla_{a} s^{a}=-\int_{\partial \mathcal{M}_{+}} \mathrm{d}^{3} x \sqrt{h_{+}}\left(u_{a}^{\mathrm{B}_{+}} s^{a}\right)-\int_{\partial \mathcal{M}_{-}} \mathrm{d}^{3} x \sqrt{h_{-}}\left(u_{a}^{\mathrm{B}_{-}} s^{a}\right) \\
+\int_{\partial \mathcal{M}_{L}} \mathrm{~d}^{3} x \sqrt{-h_{L}}\left(u_{a}^{\mathrm{B}_{\mathrm{L}}} s^{a}\right) .
\end{array}
$$

But, the surface integrals are precisely those we wrote down before in eq. (38) and eq. (39) and so we find

$$
\begin{aligned}
\Delta N^{\mathrm{x}} & =\int_{\mathcal{M}} \mathrm{d}^{4} x \sqrt{-g} \nabla_{a} n_{\mathrm{x}}^{a}=\int_{\mathcal{M}} \mathrm{d}^{4} x \sqrt{-g} \Gamma_{\mathrm{x}}, \\
\Delta S & =\int_{\mathcal{M}} \mathrm{d}^{4} x \sqrt{-g} \nabla_{a} s^{a}=\int_{\mathcal{M}} \mathrm{d}^{4} x \sqrt{-g} \Gamma_{\mathrm{s}} .
\end{aligned}
$$

These are not new results, but they serve the purpose here of establishing a direct link between global and local variables, which we will use to formulate some aspects of the non-dissipative limit of our formalism.

Consider an idealized situation of a spacetime region $\mathcal{M}$ sub-divided into a region $\mathcal{M}_{A}$ for which $\Delta N_{A}^{\mathrm{x}}<0$ and $\Delta S_{A}<0$, and another region $\mathcal{M}_{B}$ for which $\Delta N_{B}^{\mathrm{x}}>0$ and $\Delta S_{B}>0$.

15 The different sign in the integrals over $\partial \mathcal{M}_{ \pm}$and $\partial \mathcal{M}_{L}$ is due to the fact that $u_{B_{ \pm}}$are timelike while $u_{B_{L}}$ is spacelike. 
The trick is that they are such that the total changes on $\mathcal{M}$ vanish:

$$
\Delta N^{\mathrm{x}}=\Delta N_{A}^{\mathrm{x}}+\Delta N_{B}^{\mathrm{x}}=0, \Delta S=\Delta S_{A}+\Delta S_{B}=0 .
$$

The point is that, even though $\Gamma_{\mathrm{x}}$ and $\Gamma_{\mathrm{s}}$ are not zero, this is an example of a global, fully general relativistic, non-dissipative system since there is no net total particle number or total entropy change. But is this realistic? Is this the kind of definition of the non-dissipative limit we are looking for? Probably not. What is more likely is that the non-dissipative limit is better understood by breaking up $\mathcal{M}$ into many small spacetime regions $\delta \mathcal{M}$, with characteristic temporal and volume scales $\delta t$ and $(\delta l)^{3}$, respectively, as illustrated in fig. 1.

Once again, let us imagine that $\delta \mathcal{M}$ is subdivided into two regions $\delta \mathcal{M}_{A}$ and $\delta \mathcal{M}_{B}$. It is conceivable that on these scales statistical fluctuations could lead to positive creation rates in one region and negative in the other. If the regions are small enough, we can assume that $\Gamma_{\mathrm{x}}$ and $\Gamma_{\mathrm{s}}$ vary slowly across them so that we can approximate the integrals for $\delta N_{\delta \mathcal{M}}^{\mathrm{x}}$ and $\delta S_{\delta \mathcal{M}}$ as

$$
\delta N_{\delta \mathcal{M}}^{\mathrm{x}} \approx \Gamma_{\mathrm{x}} \delta t(\delta l)^{3}, \delta S_{\delta \mathcal{M}} \approx \Gamma_{\mathrm{s}} \delta t(\delta l)^{3}
$$

However, the random nature of statistical fluctuations for a system purported to be in equilibrium implies that any non-zero creation rates inside $\delta \mathcal{M}_{A}$ and $\delta \mathcal{M}_{B}$ must balance on average so that

$$
\begin{gathered}
\delta N_{\delta \mathcal{M}}^{\mathrm{x}}=\delta N_{\delta \mathcal{M}_{A}}^{\mathrm{x}}+\delta N_{\delta \mathcal{M}_{B}}^{\mathrm{x}} \approx\left(\Gamma_{\mathrm{x}}^{A}+\Gamma_{\mathrm{x}}^{B}\right) \delta t(\delta l)^{3}=0 \Longrightarrow \Gamma_{\mathrm{x}}=\Gamma_{\mathrm{x}}^{A}+\Gamma_{\mathrm{x}}^{B}=0 \\
\delta S_{\delta \mathcal{M}}=\delta S_{\delta \mathcal{M}_{A}}+\delta S_{\delta \mathcal{M}_{B}} \approx\left(\Gamma_{\mathrm{s}}^{A}+\Gamma_{\mathrm{s}}^{B}\right) \delta t(\delta l)^{3}=0 \Longrightarrow \Gamma_{\mathrm{s}}=\Gamma_{\mathrm{s}}^{A}+\Gamma_{\mathrm{s}}^{B}=0
\end{gathered}
$$

One conclusion from this exercise is that the characteristic time and volume scales of $\delta \mathcal{M}$ must be large enough that statistical fluctuations will, on average, balance out for a system in equilibrium. The second conclusion is that having $\delta N_{\delta \mathcal{M}}^{\mathrm{x}}=0\left(\delta S_{\delta \mathcal{M}}=0\right)$ on the one hand means $\Gamma_{\mathrm{x}}=0\left(\Gamma_{\mathrm{s}}=0\right)$ on the other, and vice versa. Putting both together we will assume that the equilibrium state for multi-fluid systems must be such that regions like $\delta \mathcal{M}$ set the scales for fluid elements and $\Gamma_{\mathrm{x}}=0$ and $\Gamma_{\mathrm{s}}=0$ everywhere in $\mathcal{M}$.

\section{E. Local Analysis of the Non-dissipative Limit}

This subsection begins where the previous one left off; that is, a necessary condition for a multi-fluid system to be in equilibrium is that the flux creation rates $\Gamma_{\mathrm{x}}$ (now including 
the entropy) vanish everywhere. We will use the field equations themselves to investigate three different ways for the action-based system to have zero particle creation rates: 1) The limit where the dissipation terms $R_{a}^{\mathrm{x}}$ and $D_{a b}^{\mathrm{x}}$ are zero, 2) the limit where the dissipation terms are non-zero but the fluid motion is such that the dissipative channels are dynamically suppressed, and 3) a combination of dynamical suppression with constraints between the dissipation terms that lead to Killing vector fields.

But before we investigate the zero-dissipation limit further, we will impose another condition which defines the equilibrium, and that is all distinct fluids are comoving - e.g. we are not considering systems with superfluid/superconducting phases, or a perfect heatconducting limit [16]. This means that there is a common four-velocity for all species, $u_{\mathrm{x}}^{a}=u_{\mathrm{e}}^{a}$. However, it is important to point out a subtlety about this comoving limit: For a multi-fluid system each species has its own evolution equation. Even in the comoving limit there are still $\mathrm{x}$ fluid equations. Now consider the field equations for a multi-species, single fluid system - as we see in eq. (37), it has only one fluid evolution equation. Therefore, the comoving limit of the multi-fluid system (x equations) is not equal to the single-fluid system (one equation). This is not an error, rather, it is a consequence of the fact that the number of independent field equations of the system is fixed by the number of independent fluids chosen before the action principle is applied.

We will now look in greater detail at multi-fluid systems where all particle fluxes are conserved and the species are comoving.

\section{Comoving System with Vanishing Dissipation Terms}

Here the non-dissipative limit is achieved by setting $R_{\mathrm{x}}^{a}=0$ and $D_{a b}^{\mathrm{x}}=0$. Since the fluids are comoving we have for the fluxes $n_{\mathrm{x}}^{a}=n_{\mathrm{x}} u_{\mathrm{e}}^{a}$, and so the four-momenta become

$$
\mu_{a}^{\mathrm{x}}=\left(\mathcal{B}_{\mathrm{x}} n_{\mathrm{x}}+\sum_{\mathrm{y} \neq \mathrm{x}} \mathcal{A}_{\mathrm{xy}} n_{\mathrm{y}}\right) u_{a}^{\mathrm{e}}=\mu_{\mathrm{x}} u_{a}^{\mathrm{e}} .
$$

The equation of motion for the $\mathrm{x}$-species is

$$
f_{a}^{\mathrm{x}}=2 n_{\mathrm{x}}^{b} \nabla_{[b} \mu_{a]}^{\mathrm{x}}=n_{\mathrm{x}} \mu^{\mathrm{x}} \dot{u}_{a}^{\mathrm{e}}+n_{\mathrm{x}}\left(u_{\mathrm{e}}^{b} u_{a}^{\mathrm{e}}+\delta_{a}^{b}\right) \nabla_{b} \mu^{\mathrm{x}}=n_{\mathrm{x}} \mu^{\mathrm{x}} \dot{u}_{a}^{\mathrm{e}}+n_{\mathrm{x}} D_{a} \mu^{\mathrm{x}}=0,
$$

where we have introduced the spatial covariant derivative - acting in directions perpendicular to $u_{\mathrm{e}}^{a}$-as $D_{a}$ and the time derivative " " $=u_{\mathrm{e}}^{a} \nabla_{a}$. For a scalar $A$ we have

$$
D_{a} A=\perp_{a}^{b} \nabla_{b} A=\left(\delta_{a}^{b}+u_{a}^{\mathrm{e}} u_{\mathrm{e}}^{b}\right) \nabla_{b} A=\nabla_{a} A+\dot{A} u_{a}^{\mathrm{e}},
$$


and for a vector

$$
D_{a} A_{b}=\perp_{a}^{c} \perp_{b}^{d} \nabla_{c} A_{d}
$$

where $\perp_{a}^{b}=\delta_{a}^{b}+u_{a}^{\mathrm{e}} u_{\mathrm{e}}^{b}$.

The first term in $f_{a}^{\mathrm{x}}$ then looks like the mass/energy per volume times the acceleration while we can show that the second one is a "pressure-like" term in the sense of being the gradient of a thermodynamic scalar. In fact, we have

$$
\frac{\partial \Lambda}{\partial n_{\mathrm{x}}}=-\left(\mathcal{B}_{\mathrm{x}} n_{\mathrm{x}}-\sum_{\mathrm{y} \neq \mathrm{x}} \mathcal{A}_{\mathrm{xy}} n_{\mathrm{y}}^{a} u_{a}^{\mathrm{x}}\right)=-\mu_{\mathrm{x}}
$$

and the sum of these terms provides the derivative of the total pressure $\Psi$ :

$$
\sum_{\mathrm{x}} n_{\mathrm{x}} D_{a} \mu^{\mathrm{x}}=D_{a}\left(\sum_{\mathrm{x}} n_{\mathrm{x}} \mu^{\mathrm{x}}+\Lambda\right)=D_{a} \Psi .
$$

It is important to note that each individual term cannot (in general) be considered as the derivative of the $\mathrm{x}$-species contribution to the total pressure. Partial pressures exist only when the various species do not interact.

Even though the comoving limit of the multi-fluid system is not the same as the single fluid, multi-species system, there is some overlap: Taking the sum over the chemical species of eq. (49) we find ${ }^{16}$

$$
(p+\varepsilon) \dot{u}_{a}^{\mathrm{e}}=-D_{a} p .
$$

This is the standard Euler form. One can show also that eq. (37) can be written in this form. This is an important self-consistency check. But because the multi-fluid comoving limit is not the same as the single fluid limit, we need to go back to the individual fluid equations of the multi-fluid system.

We can rewrite the individual equations of motion as

$$
\dot{u}_{b}^{\mathrm{e}}=-D_{b}\left(\log \mu_{\mathrm{x}}\right)
$$

thus, for each combination of $\mathrm{x} \neq \mathrm{y}$,

$$
D_{a}\left(\log \mu_{\mathrm{x}}\right)=D_{a}\left(\log \mu_{\mathrm{y}}\right) \Longrightarrow D_{a}\left(\log \frac{\mu_{\mathrm{x}}}{\mu_{\mathrm{y}}}\right)=0 .
$$

\footnotetext{
${ }^{16}$ We have used the standard Euler relation $\sum_{\mathrm{x}} n_{\mathrm{x}} \mu^{\mathrm{x}}=p+\varepsilon$, where $p, \varepsilon$ are the equilibrium pressure and energy density, respectively.
} 
This self-consistency therefore requires the various chemical potentials $\mu_{\mathrm{x}}$ and $\mu_{\mathrm{y}}$ (as functions on spacetime) to be proportional to each other by some factor $C_{\mathrm{y}}^{\mathrm{x}}$, which is constant in the spatial directions; namely,

$$
\mu_{\mathrm{x}}=C_{\mathrm{y}}^{\mathrm{x}} \mu_{\mathrm{y}}, D_{a} C_{\mathrm{y}}^{\mathrm{x}}=0
$$

This is to be contrasted with the single-fluid case, where there is no such restriction - in the sense of being forced by the evolution equation - between the chemical potentials. Usually, one must pose additional information. For example, for neutron stars one typically imposes that beta decay and inverse beta decay are in equilibrium.

\section{Dynamical Suppression of Dissipation}

Now consider the structure of a multi-fluid system in the comoving limit with $R_{a}^{\mathrm{x}} \neq 0$ and $D_{a b}^{\mathrm{x}} \neq 0$. This can be achieved if the fluid flow is such that the dissipation mechanisms are not triggered. We still have in place the condition that every species (including the entropy) is to be conserved $\left(\Gamma_{\mathrm{x}}=0\right)$. This implies via eq. (16) that

$$
\mu_{\mathrm{x}} \Gamma_{\mathrm{x}}=-R_{a}^{\mathrm{x}} u_{\mathrm{e}}^{a}-D_{a b}^{\mathrm{x}} \nabla^{a} u_{\mathrm{e}}^{b}=0 \Longrightarrow R_{a}^{\mathrm{x}} u_{\mathrm{e}}^{a}=-D_{a b}^{\mathrm{x}} \nabla^{a} u_{\mathrm{e}}^{b},
$$

where we have used the identity $u_{\mathrm{x}}^{b} D_{a b}^{\mathrm{x}}=0$. The total dissipation tensor $D_{a b}=\sum_{\mathrm{x}} D_{a b}^{\mathrm{x}}$ is symmetric and such that $u_{\mathrm{e}}^{b} D_{a b}=0$ automatically. If we now add eq. (58) over all species we find

$$
\sum_{\mathrm{x}} \mu_{\mathrm{x}} \Gamma_{\mathrm{x}}=-\left(\sum_{\mathrm{x}} R_{a}^{\mathrm{x}}\right) u_{\mathrm{e}}^{a}-D_{a b} \nabla^{a} u_{\mathrm{e}}^{b}=-D_{a b} D^{(a} u_{\mathrm{e}}^{b)}=0,
$$

where we have used the identity $\sum_{\mathrm{x}} R_{a}^{\mathrm{x}}=0$ and the fact that $D_{a b}$ is purely spatial with respect to $u_{\mathrm{e}}^{a}$.

Using the standard decomposition

$$
\begin{aligned}
\nabla_{a} u_{b} & =-a_{b} u_{a}+\varpi_{a b}+\sigma_{a b}+\frac{1}{3} \theta \perp_{a b}, \\
\varpi_{a b} & =\perp_{[a}^{c} \perp_{b]}^{d} \nabla_{c} u_{d}, \\
\sigma_{a b} & =\perp_{(a}^{c} \perp_{b)}^{d} \nabla_{c} u_{d}-\frac{1}{3} \theta \perp_{a b}, \\
\theta & =\nabla_{a} u^{a}=D_{a} u^{a},
\end{aligned}
$$

it is easy to see that eq. (59) implies

$$
D_{(a} u_{b)}^{\mathrm{e}}=\perp_{(a}^{c} \perp_{b)}^{d} \nabla_{c} u_{d}^{\mathrm{e}}=\nabla_{(a} u_{b)}^{\mathrm{e}}+u_{(a}^{\mathrm{e}} \dot{u}_{b)}^{\mathrm{e}}=\sigma_{a b}^{\mathrm{e}}+\frac{1}{3} \theta_{\mathrm{e}} \perp_{a b}=0,
$$


where the attached "e" means a term is evaluated with respect to $u_{\mathrm{e}}^{a}$. In particular, this tells us that the (dynamically-suppressed) equilibrium flow has zero expansion $\theta_{\mathrm{e}}=0$, and zero shear $\sigma_{a b}^{\mathrm{e}}=0$. What is left of the motion is captured by

$$
\nabla_{a} u_{b}^{\mathrm{e}}=\varpi_{a b}^{\mathrm{e}}-\dot{u}_{b}^{\mathrm{e}} u_{a}^{\mathrm{e}}
$$

which is consistent with rigid rotation.

From the definition of the creation rates, we can now write

$$
\Gamma_{\mathrm{x}}=\nabla_{a} n_{\mathrm{x}}^{a}=\dot{n}_{\mathrm{x}}+n_{\mathrm{x}} \theta_{\mathrm{e}}=\dot{n}_{\mathrm{x}}=0
$$

Assuming a thermodynamic relation in the standard way, namely that at equilibrium the energy functional of the system is $\varepsilon=\varepsilon\left(n_{\mathrm{x}}\right)$, we see that the chemical potential of each species is $\mu_{\mathrm{x}}=\mu_{\mathrm{x}}\left(n_{\mathrm{x}}\right)$ and likewise for the pressure $p$. Therefore, we have $\dot{\mu}_{\mathrm{x}}=0$ and $\dot{p}=0$, as well.

The final condition required for dynamical suppression to work comes from the equation of motion for each species; namely,

$$
\dot{u}_{a}^{\mathrm{e}}=-\nabla^{b}\left[\log \left(\mu_{\mathrm{x}}\right) g_{b a}+D_{b a}^{\mathrm{x}}\right]+R_{a}^{\mathrm{x}},
$$

which implies for all combinations of $\mathrm{x}$ and $\mathrm{y}$ that

$$
R_{a}^{\mathrm{x}}-R_{a}^{\mathrm{y}}=\nabla^{b}\left[\log \left(\mu_{\mathrm{x}} / \mu_{\mathrm{y}}\right) g_{b a}+D_{b a}^{\mathrm{x}}-D_{b a}^{\mathrm{y}}\right], \mathrm{x} \neq \mathrm{y}
$$

\section{Dynamical Suppression and Killing Vectors}

In a local region of spacetime, freely falling frames exist and the Killing equation will be satisfied approximately. In these local regions having an equilibrium will be consistent with the existence of Killing fields. However, local regions which are far removed from each other will not be (on the relevant dynamical timescale) in equilibrium with each other. This kind of "quasi-local" regression towards equilibrium has been discussed in the work of Fukuma and Sakatani [30], where the authors introduce explicitly two different spacetime scales to describe the evolution of general relativistic dissipative systems. The hypothesis of Local Thermodynamic Equilibrium applies on the smaller scale - which is of the size of the fluid

element - while the regression (in the sense of Onsager [10]) towards equilibrium takes place on the bigger one, which can still be smaller than the body size. 
A relation between the perfect fluid four-velocity and Killing vectors, for stationary axially symmetric rotating stars ${ }^{17}$ has been discussed by Gourgoulhon [31]. A similar discussion about thermodynamic equilibrium in general relativity and the existence of Killing vectors was carried out by Becattini [32]. Specifically, he shows that there must be global Killing vector fields if the total entropy of the system is to be independent of the spacelike hypersurface over which the integration is performed. As for the work presented here, we will now show how the combination $\xi_{\mathrm{x}}^{a}=\mu_{\mathrm{x}}^{-1} u_{\mathrm{e}}^{a}$ can be turned into Killing vector fields.

Using eq. (64) it can be seen that

$$
\begin{aligned}
\nabla_{a} \xi_{b}^{\mathrm{x}}+\nabla_{b} \xi_{a}^{\mathrm{x}} & =\frac{2}{\mu_{\mathrm{x}}} \nabla_{(a} u_{b)}^{\mathrm{e}}-\frac{2}{\mu_{\mathrm{x}}} u_{(a}^{\mathrm{e}}\left[\nabla_{b)} \log \left(\mu_{\mathrm{x}}\right)\right] \\
& =\frac{2}{\mu_{\mathrm{x}}}\left[\nabla_{(a} u_{b)}^{\mathrm{e}}+\dot{u}_{(a}^{\mathrm{e}} u_{b)}^{\mathrm{e}}\right]+\frac{2}{\mu_{\mathrm{x}}} u_{(a}^{\mathrm{e}}\left[\nabla^{c} D_{|c| b)}^{\mathrm{x}}-R_{b)}^{\mathrm{x}}\right] \\
& =\frac{2}{\mu_{\mathrm{x}}} u_{(a}^{\mathrm{e}}\left[\nabla^{c} D_{|c| b)}^{\mathrm{x}}-R_{b)}^{\mathrm{x}}\right] .
\end{aligned}
$$

We have already seen in eq. (58) that dynamical suppression leads to $R_{a}^{\mathrm{x}} u_{\mathrm{e}}^{a}=-D_{a b}^{\mathrm{x}} \nabla^{a} u_{\mathrm{e}}^{b}$. Clearly, if in addition we now have

$$
u_{(a}^{\mathrm{e}}\left[\nabla^{c} D_{|c| b)}^{\mathrm{x}}-R_{b)}^{\mathrm{x}}\right]=0
$$

then the $\xi_{\mathrm{x}}^{a}$ will be a global timelike Killing vector field, along which the local thermodynamical parameters $n_{\mathrm{x}}, \mu^{\mathrm{x}}, \epsilon$, and $p$ become constants of motion.

\section{F. A Final Comment on Equilibrium}

Before leaving this section we will come full circle and consider again the change in total entropy given by eq. (40). It only references spacelike hypersurfaces as part of the ad hoc choice of the boundary of the spacetime region for which the entropy change is being determined. There are no restrictions placed on the spacetime geometry in this construct; in particular, no requirement of global Killing vectors.

As a matter of practice, the change in entropy of a system is clearly dependent on its spatial size and the amount of time it has had to evolve. Couple that with the fact that a

\footnotetext{
${ }^{17}$ Note that Gourgoulhon [31] works with the enthalpy per particle instead of chemical potentials. However, this makes no difference for barotropic perfect fluids.
} 
separation of space from time in spacetime is always a choice - an arbitrary spacetime has no preferred directions, no natural "moments-of-time" — and we see that the ad hoc nature of the boundary in eq. (40) is not a drawback. It is precisely the freedom needed in order for it to incorporate a system's spatial extent and evolution time, and the fact that a separation of space from time in spacetime is always a choice.

The main reason why this is intriguing, is the Second Law of Thermodynamics only refers to the change in total entropy, not the exact value of entropy itself at moments of time (i.e. spacelike hypersurfaces). It may be that questions of equilibrium are not to be settled by the "moment-to-moment" behaviour of three-dimensional integrals, but rather by global statements of the type eq. (40) represents. This is something that we are currently investigating and hope to give more detail on in a future work.

\section{PERTURBATIONS WITH RESPECT TO EQUILIBRIUM}

With the equations of motion obtained from an action principle, we can consider perturbations away from equilibrium configurations (of the types described above) in a way that is closely related - at least from the formal perspective - to standard hydrodynamic perturbation theory. The general picture valid for Lagrangian perturbation theory is perhaps best described by Friedman and Schutz [33]. Roughly speaking, the evolution equations for the perturbed fields can be obtained by perturbing the equations obtained from the action. It is also clear - at least in principle - how to construct a Lagrangian whose variation gives the perturbed equations (see $\S 2$ of [33]). However, since we are not focussing on a stability analysis of fluid oscillations we will not consider this additional aspect here.

To set the stage for the perturbative expansion, we consider the family of worldlines (not necessarily geodesics) that each constituent of a multifluid system traces out in spacetime. Our definition of equilibrium includes the assumption that all species are comoving. Therefore, our fiducial set of worldlines representing equilibrium are those the system would have followed if it were comoving throughout its history. This then allows us to view each of the "real" worldlines $x_{\mathrm{f}}^{a}(\bar{\tau})$ as a curve in spacetime which is close to the equilibrium one $x_{\mathrm{e}}^{a}(\tau)$, with $\bar{\tau}$ and $\tau$ being the proper times of their respective curves. (See fig. 2 for an illustration of the idea.) The unit four-velocities associated with the two worldlines are

$$
u_{\mathrm{f}}^{a}=\frac{d x_{\mathrm{f}}^{a}}{d \bar{\tau}}, \quad u_{\mathrm{e}}^{a}=\frac{d x_{\mathrm{e}}^{a}}{d \tau} .
$$




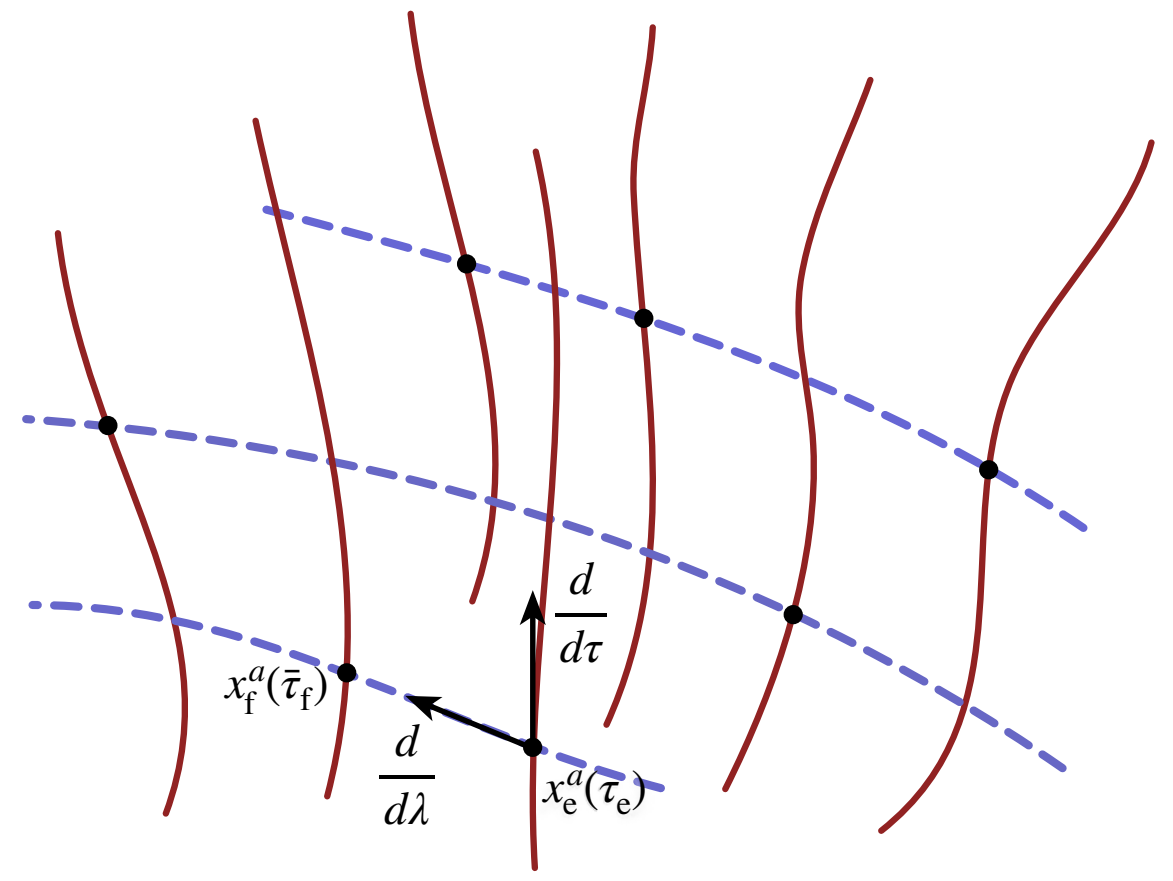

Fig. 2. An illustration of worldlines due to the fluid elements (solid vertical red lines, parameterized by $\tau, \bar{\tau}$ ) and Lagrangian displacements which connect fluid elements (dashed horizontal blue lines, parameterized by $\lambda$ ).

Obviously, $u_{\mathrm{e}}^{a}$ represents the comoving frame introduced earlier.

We assume that another family of curves $x_{\mathrm{ef}}^{a}(\lambda)$, where $\lambda$ is an affine parameter (say, the proper length), connects the equilibrium worldline to the actual one. This means that for any point $x_{\mathrm{e}}^{a}\left(\tau_{e}\right)$ on the equilibrium worldline, there is a unique point $x_{\mathrm{f}}^{a}\left(\bar{\tau}_{f}\right)$ on the perturbed worldline, and a unique curve $x_{\mathrm{ef}}^{a}(\lambda)$ between them having two points $x_{\mathrm{ef}}^{a}\left(\lambda_{\mathrm{e}}\right)$ and $x_{\mathrm{ef}}^{a}\left(\lambda_{\mathrm{f}}\right)$ such that

$$
x_{\mathrm{f}}^{a}\left(\bar{\tau}_{\mathrm{f}}\right)=x_{\text {ef }}^{a}\left(\lambda_{\mathrm{f}}\right), \quad x_{\mathrm{e}}^{a}\left(\tau_{\mathrm{e}}\right)=x_{\text {ef }}^{a}\left(\lambda_{\mathrm{e}}\right) .
$$

Taylor expanding the perturbed worldline about the equilibrium up to the second order, we get

$$
\begin{aligned}
x_{\mathrm{f}}^{a}\left(\bar{\tau}_{\mathrm{f}}\right) & =x_{\mathrm{e}}^{a}\left(\tau_{\mathrm{e}}\right)+\left.\frac{d x_{\mathrm{ef}}^{a}}{d \lambda}\right|_{\lambda_{\mathrm{e}}}\left(\lambda_{\mathrm{f}}-\lambda_{\mathrm{e}}\right)+\left.\frac{1}{2} \frac{d^{2} x_{\mathrm{ef}}^{a}}{d^{2} \lambda}\right|_{\lambda_{\mathrm{e}}}\left(\lambda_{\mathrm{f}}-\lambda_{\mathrm{e}}\right)^{2} \\
& =x_{\mathrm{e}}^{a}\left(\tau_{\mathrm{e}}\right)+\zeta^{a} \Delta \lambda+\frac{1}{2}\left(\zeta^{b} \partial_{b} \zeta^{a}\right) \Delta \lambda^{2},
\end{aligned}
$$

where we introduced the tangent vector

$$
\frac{d}{d \lambda}=\left.\frac{d x_{\mathrm{ef}}^{a}}{d \lambda}\right|_{\lambda_{\mathrm{e}}} \frac{\partial}{\partial x^{a}}=\zeta^{a} \partial_{a}
$$


The first things we want to perturb are the fluid element "names". That is, we attach a label $X^{A}$, where the index $A=1,2,3$, to each of the worldlines used to cover the region of spacetime occupied by the fluid. By definition of the Lagrangian variation $[25,33]$ we have

$$
\Delta X^{A}=\left(\phi_{*} X^{A}\left(x_{\mathrm{f}}\right)\right)\left(x_{\mathrm{e}}\right)-\bar{X}^{A}\left(x_{\mathrm{e}}\right)=X^{A}\left(x_{\mathrm{f}}\right)-\bar{X}^{A}\left(x_{\mathrm{e}}\right)=0,
$$

where $\phi$ is the diffeomorphism that connects the perturbed and unperturbed worldlines, via the flow lines $x_{\mathrm{ef}}^{a}$, and the last equality follows from the fact that the label does not change as we follow it. As a result we have, to first order

$$
\delta X^{A}=-\mathcal{L}_{\xi_{\mathrm{x}}} X^{A}=-\xi_{\mathrm{x}}^{a} \Psi_{\mathrm{e} a}^{A}=-\xi_{\mathrm{x}}^{A}
$$

where we introduced the Lagrangian displacement vector $\xi_{\mathrm{x}}^{a}=x_{\mathrm{f}}^{a}-x_{\mathrm{e}}^{a}$.

It is important to note that these displacement vectors are different from the ones introduced when obtaining the equations of motion from an action principle (see eq. (11)), even though the mathematics appears the same. In the present case the displacement vector connects two configurations that are "close" in the space of physical solutions (i.e. the second set mentioned at the beginning of section II B) - in field-theory parlance they are both "on-shell". We also note that, to compute the second order variation we cannot rely on the simple relation that exists between Lagrangian and Eulerian variation (at first order). We need to perform the calculation explicitly.

At this point, it is worth pausing to consider what is behind the perturbation scheme we are building. Since we assume the existence of a well defined equilibrium timelike congruence $x_{\mathrm{e}}^{a}$ with four velocity $u_{\mathrm{e}}^{a}$, we may imagine riding along with the equilibrium fluid element observing the evolution of the system (towards equilibrium) from this perspective. This means that the x-species four-velocity $u_{\mathrm{x}}^{a}$ can be decomposed (in the usual way) as

$$
u_{\mathrm{x}}^{a}=\gamma_{\mathrm{x}}\left(u_{\mathrm{e}}^{a}+w_{\mathrm{x}}^{a}\right), \text { where } \quad w_{\mathrm{x}}^{a} u_{a}^{\mathrm{e}}=0, \quad \gamma_{\mathrm{x}}=\left(1-w_{\mathrm{x}}^{a} w_{a}^{\mathrm{x}}\right)^{-1 / 2} .
$$

Moreover, since we are working up to first order we have

$$
\gamma_{\mathrm{x}}=1+\frac{1}{2} w_{\mathrm{x}}^{2} \approx 1+\mathcal{O}(2) \quad \Longrightarrow \quad u_{\mathrm{x}}^{a}=u_{\mathrm{e}}^{a}+w_{\mathrm{x}}^{a}
$$

We note that this linear expansion in the relative velocities, although in a different spirit, has also been discussed in the context of extensions to magneto-hydrodynamics [34-36].

Also, it is interesting in itself (and necessary for perturbing the full set of fluid equations) to understand the relation between the spatial velocity $w_{\mathrm{x}}^{a}$ as measured by the equilibrium 
observer and the Lagrangian displacement $\xi_{\mathrm{x}}^{a}$. We consider the displacement to live in the local present of the equilibrium observer, i.e., to be such that $\xi_{\mathrm{x}}^{a} u_{a}^{\mathrm{e}}=\zeta_{\mathrm{x}}^{a} u_{a}^{\mathrm{e}}=0{ }^{18}$ This implies that the vectors $\xi_{\mathrm{x}}^{a}$ and $\zeta_{\mathrm{x}}^{a}$ are spacelike non-null vector fields over the spacetime. As a result, if we consider the proper time of the perturbed worldline, we have

$$
-d \bar{\tau}^{2}=g_{a b} d x_{\mathrm{f}}^{a} d x_{\mathrm{f}}^{b}=g_{a b} d x_{\mathrm{e}}^{a} d x_{\mathrm{e}}^{b}+g_{a b}\left(d x_{\mathrm{e}}^{a} \zeta^{b} \Delta \lambda+d x_{\mathrm{e}}^{b} \zeta^{a} \Delta \lambda\right)=-d \tau^{2}
$$

where we used the fact that

$$
x_{\mathrm{e}}^{a}=x_{\mathrm{e}}^{a}(\tau) \Longrightarrow d x_{\mathrm{e}}^{a}=u_{\mathrm{e}}^{a} d \tau
$$

As a consequence, the proper time of the perturbed and equilibrium worldline is the same, so we have

$$
u_{\mathrm{x}}^{a}=\frac{d x_{\mathrm{f}}^{a}}{d \bar{\tau}} \approx \frac{d x_{\mathrm{e}}^{a}}{d \tau}+\frac{d}{d \tau} \xi_{\mathrm{x}}^{a}=u_{\mathrm{e}}^{a}+\dot{\xi}_{\mathrm{x}}^{a}
$$

where (again) the dot represents the covariant directional derivative in the direction of the equilibrium four-velocity. ${ }^{19}$ We observe that from the construction we have $w_{\mathrm{x}}^{a}=\dot{\xi}_{\mathrm{x}}^{a}$ and it is clear that when pushing the expansion to second order the relation between the two will become more involved - both because the difference between the proper times ( $\bar{\tau}$ versus $\tau$ ) appears at second order and because the Taylor expansion gets more complicated.

We now aim to understand how to construct the expansion directly in matter space. We start by noting that, since we are considering each displacement $\xi_{\mathrm{x}}^{a}$ to be orthogonal to $u_{\mathrm{e}}^{a}$ there is no loss of information in projecting the Lagrangian displacements onto the equilibrium matter space and dealing with $\xi_{\mathrm{x}}^{A}$. The general picture is thus as follows: in the general non-linear theory each matter space can be considered as an independent but interacting manifold, but this changes when we consider a perturbative expansion. In fact, the fundamental assumption of perturbation theory is that the two configurations (perturbed and unperturbed) are related by some diffeomorphism. This implies that the perturbed and unperturbed matter spaces ${ }^{20}$ are diffeomorphic, that is they are the same abstract manifold.

18 This is essentially a gauge choice, see [37] for discussion.

19 To be more precise, one should distinguish between $\frac{d}{d \tau}=u_{\mathrm{e}}^{b} \partial_{b}$ and $\frac{D}{D \tau}=u_{\mathrm{e}}^{b} \nabla_{b}$. Since we are introducing a decomposition of a vector as a sum of two, $\dot{\xi}_{\mathrm{x}}^{a}$ must be a vector as well so that the dot represents a covariant directional derivative.

${ }^{20}$ Let us recall that the matter space is obtained by taking the quotient of the spacetime over the corresponding worldline, i.e. identifying the worldline as a single point. 
Therefore we can use the same chart on the two manifolds $X^{A}$ (label the worldlines in the same way) and the difference will be only in that $X_{\mathrm{x}}^{A}\left(x^{a}\right) \neq X_{\mathrm{e}}^{A}\left(x^{a}\right)$. The difference between the two will be exactly what we found above, namely $-\xi_{\mathrm{x}}^{A}$. We also note that, by our definition of the unperturbed state, all the perturbed matter spaces are diffeomorphic to the same unperturbed one, and thus to each other.

Given this, we can work out how a general matter space tensor transforms under diffeomorphisms [24]. For instance, if we consider the projected metric $g_{\mathrm{x}}^{A B}$ we have ${ }^{21}$

$$
\delta g_{\mathrm{x}}^{A B}=-\mathcal{L}_{-\xi_{\mathrm{x}}} g_{\mathrm{x}}^{A B}=\mathcal{L}_{\xi_{\mathrm{x}}} g_{\mathrm{x}}^{A B}=\xi_{\mathrm{x}}^{C} \partial_{C} g_{\mathrm{e}}^{A B}-g_{\mathrm{e}}^{C B} \partial_{C} \xi_{\mathrm{x}}^{A}-g_{\mathrm{e}}^{A C} \partial_{C} \xi_{\mathrm{x}}^{B}
$$

where the partial derivatives are taken with respect to the equilibrium matter space coordinates. We now observe that, considering $\xi_{\mathrm{x}}^{A}$ as a scalar field in spacetime we can write

$$
-g_{\mathrm{e}}^{C B} \partial_{C} \xi^{A}=-g^{a b} \Psi_{\mathrm{e} a}^{C} \Psi_{\mathrm{e} b}^{A} \partial_{C} \xi_{\mathrm{x}}^{A}=-\Psi_{\mathrm{e} a}^{A} \nabla^{a} \xi_{\mathrm{x}}^{A}
$$

We also note that, since ${ }^{22} \partial_{C} \Psi_{\mathrm{e} a}^{A}=\partial_{a} \delta_{C}^{A}=0$, we have

$$
\partial_{C} g_{\mathrm{e}}^{A B}=2 g^{a b}\left(\frac{\partial}{\partial X_{\mathrm{e}}^{C}} \Psi_{\mathrm{e} a}^{A}\right) \Psi_{\mathrm{e} b}^{B}=0 .
$$

As a result, the projected metrics transform as

$$
\delta g_{\mathrm{x}}^{A B}=-\Psi_{\mathrm{e} a}^{B} \nabla^{a} \xi_{\mathrm{x}}^{A}-\Psi_{\mathrm{e} a}^{A} \nabla^{a} \xi_{\mathrm{x}}^{B}
$$

This also tells us that building the variation of the metric tensor in this way, we are only comparing the difference in the position of the particles, keeping fixed the spacetime metric.

We can now use the definition in eq. (50) to decompose the displacement gradients as

$$
\nabla_{a} \xi_{\mathrm{x}}^{A}=-w_{\mathrm{x}}^{A} u_{a}^{\mathrm{e}}+D_{a} \xi_{\mathrm{x}}^{A}
$$

and rewrite

$$
\begin{aligned}
\delta g_{\mathrm{x}}^{A B} & =\Psi_{\mathrm{e} a}^{B}\left(w_{\mathrm{x}}^{A} u_{\mathrm{e}}^{a}-D^{a} \xi_{\mathrm{x}}^{A}\right)+\Psi_{\mathrm{e} a}^{A}\left(w_{\mathrm{x}}^{B} u_{\mathrm{e}}^{a}-D^{a} \xi_{\mathrm{x}}^{B}\right) \\
& =-D^{B} \xi_{\mathrm{x}}^{A}-D^{A} \xi_{\mathrm{x}}^{B}
\end{aligned}
$$

\footnotetext{
${ }^{21}$ For the Lie derivative we use the formula with partial derivatives in order to avoid the possible confusion arising from the choice of the connection used on the matter space.

${ }^{22}$ If this is not immediately convincing one can prove it by taking the explicit definition of a derivative on the coordinate functions $X^{A}(\bar{X})=\delta_{C}^{A} \bar{X}^{C}=\bar{X}^{A}$ and using the linearity of the derivative.
} 
where we introduced the short-hand notation $D^{A}=\Psi_{\mathrm{e} b}^{A} g^{a b} D_{a}$. It is worth noting that this is not a strain-rate tensor of the type usually introduced in fluid dynamics, because it involves gradients in the displacements instead of velocities. The usual strain rate tensor is in fact ${ }^{23}$

$$
\begin{aligned}
\dot{g}_{\mathrm{x}}^{A B} & =-2 \Psi_{\mathrm{x}(a}^{A} \Psi_{\mathrm{x} b)}^{B}\left[-u_{\mathrm{x}}^{b} \dot{u}_{\mathrm{x}}^{a}+\varpi_{\mathrm{x}}^{a b}+\sigma_{\mathrm{x}}^{a b}+\frac{1}{3} \theta_{\mathrm{x}} \perp_{\mathrm{x}}^{a b}\right]= \\
& =-2 \Psi_{\mathrm{e}(a}^{A} \Psi_{\mathrm{e} b)}^{B}\left(\sigma_{\mathrm{e}}^{a b}+\frac{1}{3} \theta_{\mathrm{e}} \perp^{a b}\right)+\mathcal{O}(2)=-2\left(\sigma_{\mathrm{e}}^{A B}+\frac{1}{3} \theta_{\mathrm{e}} g_{\mathrm{e}}^{A B}\right),
\end{aligned}
$$

We will comment on the implications of this difference later.

Even if it is not entirely obvious what kind of object the mixed projected metric $g_{\mathrm{xy}}^{A B}$ is in the general non-linear case, in the context of a perturbative expansion there is no real difference between the various matter spaces (they are all diffeomorphic to the equilibrium one). This means that we can use the same fundamental formula also for $g_{\mathrm{xy}}$ to get

$$
\begin{aligned}
\delta g_{\mathrm{xy}}^{A B} & =g_{\mathrm{xy}}^{A B}-g_{\mathrm{e}}^{A B}=g^{a b}\left(\delta \Psi_{\mathrm{x} a}^{A} \Psi_{\mathrm{e} b}^{B}+\delta \Psi_{\mathrm{y} b}^{B} \Psi_{\mathrm{e} a}^{A}\right)= \\
& =-\Psi_{\mathrm{e} a}^{B} \nabla^{a} \xi_{\mathrm{x}}^{A}-\Psi_{\mathrm{e} a}^{A} \nabla^{a} \xi_{\mathrm{y}}^{B} .
\end{aligned}
$$

It is interesting to note that since $\delta g^{a b}=0$ we have

$$
\left[\delta, \nabla_{a}\right]=\left[\delta, \partial_{a}\right]=0
$$

That is, the variation commutes with both partial and covariant derivatives. This will become relevant when we need to work out the variation of the reactive terms that stem from a dependence of the $\mathcal{N}_{\mathrm{x}}$ on $g_{\mathrm{xy}}^{A B}$ and $g_{\mathrm{y}}^{A B}$.

There has been a number of recent efforts on building first-order dissipative hydrodynamic models starting from a field-theory perspective. It makes sense to point out the differences between the present expansion and the field-theory-based ones. From a field theory perspective hydrodynamics is the low-energy limit of a more fundamental theory. Starting from this point of view, different authors have proposed (see, for example, [17, 18]) to introduce dissipation in the models through a gradient expansion. Practically, this boils down to postulating the most general constitutive equations - that is the relations between thermodynamic forces and fluxes - in terms of the standard hydrodynamic variables (like $T, \mu \ldots)$ and their derivatives. In this context, the models are said to be of first order if the constitutive equations involve all permissible terms with just one derivative. When the system is close to equilibrium one can expect the gradients in temperature, chemical potential

23 To see this one has to use $\mathcal{L}_{u_{\mathrm{x}}} \Psi_{\mathrm{x} a}^{A}=0$. 
etc... to be small, so that terms with two or more derivatives are dominated by first-order ones. The final aim is (again) to obtain a set of equations valid close to equilibrium.

In the present work, the variables that define the physical state of the system take values close to the equilibrium ones, and by "first order" we mean the deviations are expanded up to $\mathcal{O}\left(\xi_{\mathrm{x}}\right)$. It is therefore clear that the present approach differs from the field-theorybased (gradient) expansions. The ultimate reason is that the action-based model provides the exact equations, which we then approximate, while in the field-theory approach one is trying to build up the full equations by successive expansions.

\section{ENERGY DENSITY MINIMIZATION AND EQUILIBRIUM}

In order to describe out-of-equilibrium systems in the Extended Irreversible Thermodynamic (EIT) paradigm [38], one postulates the existence of a generalized entropy-a function of a larger set of Degrees of Freedom than the corresponding equilibrium oneswhich is maximized at equilibrium. The starting point of the formalism used here is that of a generalized energy where the only degrees of freedom are the fluxes. The action-based model provides the total stress-energy-momentum tensor $T_{a b}$ of the system, so that we can easily extract the total energy density $\epsilon$ for some observer having four-velocity $u^{a}$ via the projection $\epsilon=u^{a} u^{b} T_{a b}$. We will show that requiring the local energy density to be at a minimum in equilibrium means the viscous stress tensors have to be zero.

When specific modeling is done, such as numerical evolutions, we would need to provide an equation of state (EoS) and specify values for the microphysical input parameters. From the phenomenological point of view, this corresponds to assuming the existence of a function - in our case, energy density - defined on some "thermodynamical manifold" whose coordinates are the relevant degrees of freedom. Practically speaking, the formalism developed here identifies the thermodynamical manifold as being the matter space used in the variational model. As the general discussion gets quite complex, we focus on the specific example of a two component system, with the components representing matter and entropy (see also $[39,40])$.

Let us first consider the non-dissipative limit. Thermodynamics of a single fluid is de- 
scribed by some equilibrium energy $\varepsilon_{\mathrm{e}}(n, s)$ such that

$$
d \varepsilon_{\mathrm{e}}=T d s+\mu d n=\sum_{\mathrm{x}=\mathrm{n}, \mathrm{s}} \mu^{\mathrm{x}} d n_{\mathrm{x}}
$$

On the other hand, the conservative variational model is built using a master function $\Lambda\left(n_{\mathrm{n}}^{2}, n_{\mathrm{s}}^{2}, n_{\mathrm{ns}}^{2}\right)$. Because of our assumption that all species are comoving while in equilibrium there is no heat flux relative to the matter and therefore $n_{\mathrm{ns}}^{2}=-g_{a b} n_{\mathrm{n}}^{a} n_{\mathrm{s}}^{b}=+n_{\mathrm{n}} n_{\mathrm{s}}$, and the master function only depends on two variables, $\Lambda=\Lambda\left(n_{\mathrm{n}}, n_{\mathrm{s}}\right)$. It is indeed easy to see that the equilibrium energy density, as measured by the equilibrium observer, is

$$
\hat{\varepsilon}_{\mathrm{e}}=T_{a b} u_{\mathrm{e}}^{a} u_{\mathrm{e}}^{b}=\left[\Psi g_{a b}+(\Psi-\Lambda) u_{a}^{\mathrm{e}} u_{b}^{\mathrm{e}}\right] u_{\mathrm{e}}^{a} u_{\mathrm{e}}^{b}=-\Lambda
$$

Since we have already identified the matter space normalizations of the three-forms with the rest frame densities $\mathcal{N}_{\mathrm{x}}=n_{\mathrm{x}}$, we can think of the thermodynamic energy as a function defined on the matter space, and write

$$
\hat{\varepsilon}_{\mathrm{e}}=\hat{\varepsilon}_{\mathrm{e}}\left(\mathcal{N}_{\mathrm{n}}, \mathcal{N}_{\mathrm{s}}\right)=-\Lambda_{\mathrm{e}}\left(\mathcal{N}_{\mathrm{n}}, \mathcal{N}_{\mathrm{s}}\right)
$$

The equilibrium case suggests that we could try to extend this identification to the nonequilibrium setting, and "build" the thermodynamics on the matter space. This raises the (difficult) question of what the global matter space is in the full non-linear case. We will not address that here. Instead, we focus on the near-equilibrium case, where we only have to deal with the equilibrium matter space.

Because of the way we have built the expansion, it is natural to project tensor quantitiesflux, stress-energy-momentum tensor, etcetera - into the frame of the equilibrium observer, as defined by the equilibrium worldlines congruence $u_{\mathrm{e}}^{a}$. Quantities measured in this frame will be indicated by a "hat". Those without a hat are measured in fluid rest frames, which are defined by the $u_{\mathrm{x}}^{a}$. The equilibrium value of a quantity in the equilibrium frame will be indicated with a "bar". For instance, the particle density measured in the equilibrium frame is $\hat{n}_{\mathrm{x}}=-u_{a}^{\mathrm{e}} n_{\mathrm{x}}^{a}$; in the $\mathrm{x}$-fluid rest frame it is $n_{\mathrm{x}}=-u_{a}^{\mathrm{x}} n_{\mathrm{x}}^{a}$; and the equilibrium value in the equilibrium frame is $\bar{n}_{\mathrm{x}}=\left.\hat{n}_{\mathrm{x}}\right|_{\mathrm{e}}$.

The "out-of-equilibrium" energy density $\hat{\varepsilon}_{\text {o.e. }}$ of the system as determined in the equilibrium rest frame is given by

$$
\hat{\varepsilon}_{\text {o.e. }}=\left(T_{\text {n.d. }}^{a b}+\sum_{\mathrm{x}} D_{\mathrm{x}}^{a b}\right) u_{a}^{\mathrm{e}} u_{b}^{\mathrm{e}}=\varepsilon_{\text {o.e. }}^{\text {n.d. }}+D^{a b} u_{a}^{\mathrm{e}} u_{b}^{\mathrm{e}},
$$


where we have separated the contribution from the viscous stress tensor $D_{a b}$ from those having the "non-dissipative" form; namely,

$$
T_{\text {n.d. }}^{a b}=\left(\Lambda-\sum_{\mathrm{x}} n_{\mathrm{x}}^{c} \mu_{c}^{\mathrm{x}}\right) g^{a b}+\sum_{\mathrm{x}} n_{\mathrm{x}}^{a} \mu_{\mathrm{x}}^{b}=\Psi g^{a b}+\sum_{\mathrm{x}} n_{\mathrm{x}}^{a} \mu_{\mathrm{x}}^{b}
$$

The expression for $\hat{\varepsilon}_{\text {o.e. }}$ can be made more explicit by means of eq. (52), which leads to $\Psi=\Lambda+\sum_{\mathrm{x}} n_{\mathrm{x}} \mu_{\mathrm{x}}$ and

$$
\hat{\varepsilon}_{\text {o.e. }}^{\text {n.d. }}=u_{a}^{\mathrm{e}} u_{b}^{\mathrm{e}} T_{\text {n.d. }}^{a b}=-\Lambda-\sum_{\mathrm{x}=n, s}\left(n_{\mathrm{x}} \mu_{\mathrm{x}}-\hat{n}_{\mathrm{x}} \hat{\mu}_{\mathrm{x}}\right)
$$

Because flux is a vector, the two densities $\hat{n}_{\mathrm{x}}$ and $n_{\mathrm{x}}$ are easily shown to be related by

$$
\hat{n}_{\mathrm{x}}=-n_{\mathrm{x}}^{a} u_{a}=-n_{\mathrm{x}} u_{\mathrm{x}}^{a} u_{a}=\left(1-w_{\mathrm{x}}^{a} w_{a}^{\mathrm{x}}\right)^{-1 / 2} n_{\mathrm{x}}=\left(1+\frac{1}{2} w_{\mathrm{x}}^{2}\right) n_{\mathrm{x}}+\mathcal{O}(3)
$$

Meanwhile, the corresponding momentum relation is a bit more involved because of the entrainment:

$$
\begin{aligned}
\mu_{\mathrm{x}} & =-\mu_{b}^{\mathrm{x}} u_{\mathrm{x}}^{b}=-\gamma_{\mathrm{x}}\left(u^{b}+w_{\mathrm{x}}^{b}\right)\left(\mathcal{B}_{\mathrm{x}} n_{\mathrm{x}} u_{b}^{\mathrm{x}}+\sum_{\mathrm{y} \neq \mathrm{x}} \mathcal{A}_{\mathrm{xy}} n_{\mathrm{y}} u_{\mathrm{y}}^{b}\right) \\
& =\gamma_{\mathrm{x}}\left(\hat{\mu}_{\mathrm{x}}-\mathcal{B}_{\mathrm{x}} n_{\mathrm{x}} \gamma_{\mathrm{x}} w_{\mathrm{x}}^{2}-\sum_{\mathrm{y} \neq \mathrm{x}} \mathcal{A}_{\mathrm{xy}} n_{\mathrm{y}} \gamma_{\mathrm{y}} w_{\mathrm{x}}^{a} w_{a}^{\mathrm{y}}\right)
\end{aligned}
$$

We can rearrange this as

$$
\hat{\mu}_{\mathrm{x}}=\mu_{\mathrm{x}}-\frac{1}{2} \bar{\mu}_{\mathrm{x}} w_{\mathrm{x}}^{2}+\overline{\mathcal{B}}_{\mathrm{x}} \bar{n}_{\mathrm{x}} w_{\mathrm{x}}^{2}+\sum_{\mathrm{y} \neq \mathrm{x}} \overline{\mathcal{A}}_{\mathrm{xy}} \bar{n}_{\mathrm{y}} w_{\mathrm{x}}^{a} w_{a}^{\mathrm{y}}
$$

and, wrapping up, we get

$$
\begin{aligned}
\hat{\varepsilon}_{\text {o.e. }}^{n . d .} & =-\Lambda+\overline{\mathcal{B}}_{\mathrm{n}} \bar{n}_{\mathrm{n}}^{2} w_{\mathrm{n}}^{2}+\overline{\mathcal{B}}_{\mathrm{s}} \bar{n}_{\mathrm{s}}^{2} w_{\mathrm{s}}^{2}+2 \overline{\mathcal{A}}_{\mathrm{ns}} \bar{n}_{\mathrm{s}} \bar{n}_{\mathrm{n}} w_{\mathrm{n}}^{a} w_{a}^{\mathrm{s}} \\
& =-\Lambda+\bar{\mu}_{\mathrm{n}} \bar{n}_{\mathrm{n}} w_{\mathrm{n}}^{2}+\bar{\mu}_{\mathrm{s}} \bar{n}_{\mathrm{s}} w_{\mathrm{s}}^{2}-\mathcal{A}_{\mathrm{ns}} \bar{n}_{\mathrm{n}} \bar{n}_{\mathrm{s}}\left(w_{\mathrm{n}}^{a}-w_{\mathrm{s}}^{a}\right)^{2} .
\end{aligned}
$$

It is now clear that, in order to proceed, we need an expansion for the master function, $\Lambda$.

Note that the dissipative action model assumes $\Lambda$ depends on $\left(X_{\mathrm{n}}^{A}, X_{\mathrm{s}}^{A}, g_{\mathrm{n}}^{A B}, g_{\mathrm{s}}^{A B}, g_{\mathrm{ns}}^{A B}\right)$ through the scalar product of the fluxes $n_{\mathrm{n}}^{2}, n_{\mathrm{s}}^{2}, n_{\mathrm{ns}}^{2}$. Therefore, in order to build the expansion we can expand $\Lambda$ up to second order in the standard way (see [37]). We thus have

$$
\begin{aligned}
\Lambda=\Lambda_{\mathrm{e}} & -\frac{1}{2} \sum_{\mathrm{x}=n, s} \mathcal{B}_{\mathrm{x}} \delta n_{\mathrm{x}}^{2}-\mathcal{A}_{\mathrm{ns}} \delta n_{\mathrm{ns}}^{2}-\frac{1}{4} \sum_{\mathrm{x}=n, s} \frac{\partial \mathcal{B}_{\mathrm{x}}}{\partial n_{\mathrm{x}}^{2}}\left(\delta n_{\mathrm{x}}^{2}\right)^{2}-\frac{1}{2} \frac{\partial \mathcal{A}_{\mathrm{ns}}}{\partial n_{\mathrm{ns}}^{2}}\left(\delta n_{\mathrm{ns}}^{2}\right)^{2} \\
& -\frac{1}{2} \frac{\partial \mathcal{B}_{\mathrm{n}}}{\partial n_{\mathrm{s}}^{2}}\left(\delta n_{\mathrm{n}}^{2}\right)\left(\delta n_{\mathrm{s}}^{2}\right)-\frac{\partial \mathcal{A}_{\mathrm{ns}}}{\partial n_{\mathrm{n}}^{2}}\left(\delta n_{\mathrm{n}}^{2}\right)\left(\delta n_{\mathrm{ns}}^{2}\right)-\frac{\partial \mathcal{A}_{\mathrm{ns}}}{\partial n_{\mathrm{s}}^{2}}\left(\delta n_{\mathrm{s}}^{2}\right)\left(\delta n_{\mathrm{ns}}\right)^{2}
\end{aligned}
$$


To make contact with the previous expansion on the matter space we need explicit expressions for $\delta n_{\mathrm{x}}^{2}$ and all other similar terms that appear in the expression above.

For the four-current we have

$$
\begin{aligned}
\delta n_{\mathrm{x}}^{a} & =n_{\mathrm{x}}^{a}-\bar{n}_{\mathrm{x}}^{a}=\left(\bar{n}_{\mathrm{x}}+\delta n_{\mathrm{x}}\right)\left[\left(1+\frac{1}{2} w_{\mathrm{x}}^{2}\right) u^{a}+w_{\mathrm{x}}^{a}\right]-\bar{n}_{\mathrm{x}} u^{a} \\
& =\frac{1}{2} \bar{n}_{\mathrm{x}} w_{\mathrm{x}}^{2} u^{a}+\bar{n}_{\mathrm{x}} w_{\mathrm{x}}^{a}+\delta n_{\mathrm{x}} u^{a}+\delta n_{\mathrm{x}} w_{\mathrm{x}}^{a}
\end{aligned}
$$

and we see that it - quite intuitively - changes both as the density and the four-velocity change. By means of eq. (99) we get

$$
\delta n_{\mathrm{x}}^{2}=-\left(2 \bar{n}_{\mathrm{x}}^{a} \delta n_{a}^{\mathrm{x}}+\delta n_{\mathrm{x}}^{a} \delta n_{a}^{\mathrm{x}}\right)=2 \bar{n}_{\mathrm{x}} \delta n_{\mathrm{x}}+\left(\delta n_{\mathrm{x}}\right)^{2}
$$

Similarly, we have

$$
\begin{aligned}
\delta n_{\mathrm{xy}}^{2} & =-\left(\bar{n}_{\mathrm{x}}^{a} \delta n_{a}^{\mathrm{y}}+\bar{n}_{\mathrm{y}}^{a} \delta n_{a}^{\mathrm{x}}+\delta n_{\mathrm{x}}^{a} \delta n_{a}^{\mathrm{y}}\right) \\
& =\bar{n}_{\mathrm{x}} \delta n_{\mathrm{y}}+\bar{n}_{\mathrm{y}} \delta n_{\mathrm{x}}+\delta n_{\mathrm{x}} \delta n_{\mathrm{y}}+\frac{1}{2} \bar{n}_{\mathrm{x}} \bar{n}_{\mathrm{y}}\left(w_{\mathrm{x}}^{a}-w_{\mathrm{y}}^{a}\right)^{2}
\end{aligned}
$$

In order to complete the second order expansion of $\Lambda$ we also need the products (for every possible combination) of eq. (100) and eq. (101). These are found to be

$$
\begin{aligned}
\left(\delta n_{\mathrm{x}}^{2}\right)^{2} & =4 \bar{n}_{\mathrm{x}}^{2}\left(\delta n_{\mathrm{x}}\right)^{2} \\
\left(\delta n_{\mathrm{xy}}^{2}\right)^{2} & =\bar{n}_{\mathrm{x}}^{2}\left(\delta n_{\mathrm{y}}\right)^{2}+\bar{n}_{\mathrm{y}}^{2}\left(\delta n_{\mathrm{x}}\right)^{2}+2 \bar{n}_{\mathrm{x}} \bar{n}_{\mathrm{y}} \delta n_{\mathrm{x}} \delta n_{\mathrm{y}} \\
\left(\delta n_{\mathrm{x}}^{2}\right)\left(\delta n_{\mathrm{y}}^{2}\right) & =4 \bar{n}_{\mathrm{x}} \bar{n}_{\mathrm{y}} \delta n_{\mathrm{x}} \delta n_{\mathrm{y}}, \\
\left(\delta n_{\mathrm{xy}}^{2}\right)\left(\delta n_{\mathrm{x}}^{2}\right) & =2 \bar{n}_{\mathrm{x}}\left(\delta n_{\mathrm{x}}\right)\left(\bar{n}_{\mathrm{y}} \delta n_{\mathrm{x}}+\bar{n}_{\mathrm{x}} \delta n_{\mathrm{y}}\right) .
\end{aligned}
$$

Plugging these expressions into eq. (98) we find (up to second order)

$$
\begin{aligned}
\hat{\varepsilon}_{\mathrm{o} . \mathrm{e} .}^{n . d .} & =\varepsilon_{\mathrm{e}}\left(\bar{n}_{\mathrm{n}}, \bar{n}_{\mathrm{s}}\right)+\bar{\mu}_{\mathrm{n}} \delta n_{\mathrm{n}}+\bar{\mu}_{\mathrm{s}} \delta n_{\mathrm{s}}+\frac{1}{2}\left(\overline{\mathcal{B}}_{\mathrm{n}} \bar{c}_{\mathrm{n}}^{2}-\overline{\mathcal{A}}_{u u}^{\mathrm{nn}}\right)\left(\delta n_{\mathrm{n}}\right)^{2} \\
& +\frac{1}{2}\left(\overline{\mathcal{B}}_{\mathrm{s}} \bar{c}_{\mathrm{s}}^{2}-\overline{\mathcal{A}}_{u u}^{\mathrm{ss}}\right)\left(\delta n_{\mathrm{s}}\right)^{2}-\left(\bar{\chi}_{u u}^{\mathrm{sn}}+\overline{\mathcal{A}}_{u u}^{\mathrm{ns}}\right)\left(\delta n_{\mathrm{n}}\right)\left(\delta n_{\mathrm{s}}\right)+\bar{\mu}_{\mathrm{n}} \bar{n}_{\mathrm{n}} w_{\mathrm{n}}^{2} \\
& +\bar{\mu}_{\mathrm{s}} \bar{n}_{\mathrm{s}} w_{\mathrm{s}}^{2}-\frac{1}{2} \overline{\mathcal{A}}_{\mathrm{ns}} \bar{n}_{\mathrm{n}} \bar{n}_{\mathrm{s}} w_{\mathrm{ns}}^{2}
\end{aligned}
$$


where we have made use of eq. (97) and defined

$$
\begin{aligned}
\bar{c}_{\mathrm{x}}^{2} & =1+2 \frac{\bar{n}_{\mathrm{x}}^{2}}{\overline{\mathcal{B}}_{\mathrm{x}}} \frac{\partial \overline{\mathcal{B}}_{\mathrm{x}}}{\partial n_{\mathrm{x}}^{2}} \\
w_{\mathrm{xy}}^{2} & =g_{a b}\left(w_{\mathrm{x}}^{a}-w_{\mathrm{y}}^{a}\right)\left(w_{\mathrm{x}}^{b}-w_{\mathrm{y}}^{b}\right) \\
\overline{\mathcal{A}}_{a b}^{\mathrm{xx}} & =-\left(\bar{n}_{\mathrm{y}}^{2} \frac{\partial \overline{\mathcal{A}}_{\mathrm{xy}}}{\partial n_{\mathrm{xy}}^{2}}+4 \bar{n}_{\mathrm{x}} \bar{n}_{\mathrm{y}} \frac{\partial \overline{\mathcal{A}}_{\mathrm{xy}}}{\partial n_{\mathrm{x}}^{2}}\right) u_{a}^{\mathrm{e}} u_{b}^{\mathrm{e}} \doteq \overline{\mathcal{A}}_{u u}^{\mathrm{xx}} u_{a}^{\mathrm{e}} u_{b}^{\mathrm{e}} \\
\overline{\mathcal{A}}_{a b}^{\mathrm{ns}} & =\overline{\mathcal{A}}^{n s} \perp_{a b}-\left(\overline{\mathcal{A}}_{\mathrm{ns}}+2 \bar{n}_{\mathrm{n}}^{2} \frac{\partial \overline{\mathcal{A}}^{\mathrm{ns}}}{\partial n_{\mathrm{n}}^{2}}+2 \bar{n}_{\mathrm{s}}^{2} \frac{\partial \overline{\mathcal{A}}^{\mathrm{ns}}}{\partial n_{\mathrm{s}}^{2}}+\bar{n}_{\mathrm{n}} \bar{n}_{\mathrm{s}} \frac{\partial \overline{\mathcal{A}}^{\mathrm{ns}}}{\partial n_{\mathrm{ns}}^{2}}\right) u_{a}^{\mathrm{e}} u_{b}^{\mathrm{e}} \\
& \doteq \overline{\mathcal{A}}^{\mathrm{ns}} \perp_{a b}+\overline{\mathcal{A}}_{u u}^{\mathrm{ns}} u_{a}^{\mathrm{e}} u_{b}^{\mathrm{e}} \\
\bar{\chi}_{u u}^{\mathrm{ns}} & =-2 \bar{n}_{\mathrm{n}} \bar{n}_{\mathrm{s}} \frac{\partial \overline{\mathcal{B}}^{\mathrm{n}}}{\partial n_{\mathrm{s}}^{2}}=-2 \bar{n}_{\mathrm{n}} \bar{n}_{\mathrm{s}} \frac{\partial \overline{\mathcal{B}}^{\mathrm{s}}}{\partial n_{\mathrm{n}}^{2}}
\end{aligned}
$$

(See Andersson and Comer [37] for more discussion of these terms and Samuelsson et al. [41] for their roles in two-stream instability.)

Noting that the quantity $\delta n_{\mathrm{x}}$ is the variation of the rest frame density, we can relate it to a variation of $\mathcal{N}_{\mathrm{x}}$ and "close the loop". Since the $\mathcal{N}_{\mathrm{x}}$ are functions on matter space of the variables $\left(X_{\mathrm{n}}, X_{\mathrm{s}}, g_{\mathrm{n}}^{A B}, g_{\mathrm{s}}^{A B}, g_{\mathrm{ns}}^{A B}\right)$ the expression for the energy is actually a second order expansion in terms of those variables. We note also that, because of the two-layer structure, the $\delta n_{\mathrm{x}}$ above contain second-order terms.

A priori, the expression in eq. (103) does not provide the total out-of-equilibrium energy because we need to account for the contributions due to dissipative terms. However, we will now show that these actually do not contribute. To do this, we assume an expansion for all the viscous stress tensors of the form

$$
S_{A B}=S_{A B}^{\mathrm{e}}+S_{A B}^{1}+S_{A B}^{2}+\mathcal{O}(3)
$$

without specifying (for now) the explicit expressions. Recalling the fact that $\Psi_{\mathrm{e} a}^{A} u_{\mathrm{e}}^{a}=0$, we can write, therefore,

$$
S_{a b} u_{\mathrm{e}}^{a} u_{\mathrm{e}}^{b}=S_{A B}\left(X_{\mathrm{e}}^{A}+\delta X^{A}\right)_{, a}\left(X_{\mathrm{e}}^{B}+\delta X^{B}\right)_{, b} u_{\mathrm{e}}^{a} u_{\mathrm{e}}^{b}=S_{A B}^{\mathrm{e}} \delta X_{, a}^{A} \delta X_{, b}^{B} u_{\mathrm{e}}^{a} u_{\mathrm{e}}^{b}
$$

where the expansion is up to second order. It is clear that this argument is valid for each viscous stress tensor, and for $D_{a b} u_{\mathrm{e}}^{a} u_{\mathrm{e}}^{b}$ as well, so that the dissipative contributions to the off-equilibrium energy are, at least, of second order. Assuming that the energy is minimized, that is

$$
\hat{\varepsilon}_{\text {o.e. }}^{n . d .}-\varepsilon_{\mathrm{e}}\left(\bar{n}_{\mathrm{n}}, \bar{n}_{\mathrm{s}}\right)=0+\mathcal{O}(2) \text {, }
$$


we then have

$$
\bar{\mu}_{\mathrm{n}} \delta n_{\mathrm{n}}+\bar{\mu}_{\mathrm{s}} \delta n_{\mathrm{s}}=\mathcal{O}(2)
$$

which has a clear thermodynamical interpretation and is consistent with the EIT picture, see [38], since, up to first order, the generalized energy is a function of the $n_{\mathrm{x}}$ only.

We want to translate the above result into conditions for the matter space functions $\mathcal{N}_{\mathrm{x}}$. We start by observing that in the conservative case, the three-form $n_{A B C}^{\mathrm{x}}$ is a function of the $X_{\mathrm{x}}^{A}$ coordinates only. Therefore, $\overline{\mathcal{N}}_{\mathrm{x}}$ is just a function of $X_{\mathrm{x}}^{A}$, while, because $\mathcal{N}_{\mathrm{x}}=\overline{\mathcal{N}}_{\mathrm{x}} \sqrt{g^{\mathrm{x}}}$, the latter depends also on the projected metric

$$
\frac{\partial \mathcal{N}_{\mathrm{x}}}{\partial g_{\mathrm{x}}^{A B}}=\frac{1}{2} \sqrt{g^{\mathrm{x}}} \overline{\mathcal{N}}_{\mathrm{x}} g_{A B}^{\mathrm{x}}=\frac{1}{2} \mathcal{N}_{\mathrm{x}} g_{A B}^{\mathrm{x}}
$$

When considering the expansion of $n_{\mathrm{x}}$ (and hence $\mathcal{N}_{\mathrm{x}}$ ) we assume that we can write

$$
\mathcal{N}_{\mathrm{x}}=\mathcal{N}_{\mathrm{x}}^{\mathrm{e}}+\mathcal{N}_{\mathrm{x}}^{\mathrm{d}}
$$

where $\mathcal{N}_{\mathrm{x}}^{\mathrm{e}}$ is the same as in the non-dissipative limit while the dissipative contribution $\mathcal{N}_{\mathrm{x}}^{\mathrm{d}}$ is a function also of the additional variables that encode the dissipation. The separation of $\mathcal{N}_{\mathrm{x}}$ into two pieces can naturally assume that $\mathcal{N}_{\mathrm{x}}^{\mathrm{d}}$ vanishes at equilibrium but not its derivatives.

Since the equilibrium evolves in a conservative fashion, we can write

$$
\begin{aligned}
\delta n_{\mathrm{x}} & \equiv \mathcal{N}_{\mathrm{x}}-\mathcal{N}_{\mathrm{x}}^{\mathrm{e}}=\mathcal{N}_{\mathrm{x}}^{\mathrm{d}}=\mathcal{N}_{\mathrm{x}}^{\mathrm{d}}-\left.\mathcal{N}_{\mathrm{x}}^{\mathrm{d}}\right|_{\mathrm{e}} \\
& =\frac{\partial \mathcal{N}_{\mathrm{x}}^{\mathrm{d}}}{\partial X_{\mathrm{x}}^{A}} \delta X_{\mathrm{x}}^{A}+\frac{\partial \mathcal{N}_{\mathrm{x}}^{\mathrm{d}}}{\partial X_{\mathrm{y}}^{A}} \delta X_{\mathrm{y}}^{A}+\frac{\partial \mathcal{N}_{\mathrm{x}}^{\mathrm{d}}}{\partial g_{\mathrm{x}}^{A B}} \delta g_{\mathrm{x}}^{A B}+\frac{\partial \mathcal{N}_{\mathrm{x}}^{\mathrm{d}}}{\partial g_{\mathrm{y}}^{A B}} \delta g_{\mathrm{y}}^{A B}+\frac{\partial \mathcal{N}_{\mathrm{x}}^{\mathrm{d}}}{\partial g_{\mathrm{xy}}^{A B}} \delta g_{\mathrm{xy}}^{A B}+\mathcal{O}(2)
\end{aligned}
$$

where here, and in similar expansions below, every quantity is to be evaluated at equilibrium. With this assumption it is easy to read off from eq. (108) the first order relation

$$
\mathcal{M}_{\mathrm{n}} d \mathcal{N}_{\mathrm{n}}^{\mathrm{d}}+\mathcal{M}_{\mathrm{s}} d \mathcal{N}_{\mathrm{s}}^{\mathrm{d}}=0
$$

This leads to

$$
\mathcal{M}_{\mathrm{n}} \frac{\partial \mathcal{N}_{\mathrm{n}}^{\mathrm{d}}}{\partial X_{\mathrm{n}}^{A}}+\mathcal{M}_{\mathrm{s}} \frac{\partial \mathcal{N}_{\mathrm{s}}^{\mathrm{d}}}{\partial X_{\mathrm{n}}^{A}}=0 .
$$

The analogous results for variations with respect to $X_{\mathrm{s}}^{A}, g_{\mathrm{n}}^{A B}, g_{\mathrm{s}}^{A B}$ and $g_{\mathrm{ns}}^{A B}$ follow immediately. In particular, this shows that the total viscous stress tensor, acting on each component $D_{a b}^{\mathrm{x}}$, vanishes when the energy is minimized.

To see this explicitly we note that (see eqs. (31) and (32))

$$
\mathcal{S}_{A B}^{\mathrm{xy}, \mathrm{e}} \equiv 2 \mathcal{M}_{\mathrm{x}} \frac{\partial \mathcal{N}_{\mathrm{x}}}{\partial g_{\mathrm{xy}}^{A B}}=-2 \mathcal{M}_{\mathrm{y}} \frac{\partial \mathcal{N}_{\mathrm{y}}}{\partial g_{\mathrm{xy}}^{A B}}=-\mathcal{S}_{B A}^{\mathrm{yx}, \mathrm{e}}
$$


where we made use of the symmetry property of the mixed metric, namely $g_{\mathrm{xy}}^{A B}=g_{\mathrm{yx}}^{B A}$. Similarly,

$$
\begin{aligned}
S_{A B}^{\mathrm{x}, \mathrm{e}} & \equiv 2 \mathcal{M}_{\mathrm{x}}\left(\frac{\partial \mathcal{N}_{\mathrm{x}}}{\partial g_{\mathrm{x}}^{A B}}-\frac{1}{2} \mathcal{N}_{\mathrm{x}} g_{A B}^{\mathrm{x}}\right) \\
& =2 \mathcal{M}_{\mathrm{x}}\left[\frac{\partial \mathcal{N}_{\mathrm{x}}^{\mathrm{d}}}{\partial g_{\mathrm{x}}^{A B}}-\frac{1}{2}\left(\mathcal{N}_{\mathrm{x}}-\mathcal{N}_{\mathrm{x}}^{\mathrm{e}}\right) g_{A B}^{\mathrm{x}}\right] \\
& =2 \mathcal{M}_{\mathrm{x}} \frac{\partial \mathcal{N}_{\mathrm{x}}^{\mathrm{d}}}{\partial g_{\mathrm{x}}^{A B}}=-2 \mathcal{M}_{\mathrm{y}} \frac{\partial \mathcal{N}_{\mathrm{y}}^{\mathrm{d}}}{\partial g_{\mathrm{x}}^{A B}}=-s_{A B}^{\mathrm{yx}, \mathrm{e}}
\end{aligned}
$$

It is now clear that, by means of eq. (114) and eq. (115), the x-species viscous stress tensor

$$
D_{A B}^{\mathrm{x}, \mathrm{e}}=S_{A B}^{\mathrm{x}, \mathrm{e}}+s_{A B}^{\mathrm{yx}, \mathrm{e}}+\frac{1}{2}\left(\mathcal{S}_{A B}^{\mathrm{xy}, \mathrm{e}}+\mathcal{S}_{B A}^{\mathrm{yx}, \mathrm{e}}\right)=0
$$

We have considered the fully general case with all the additional dependences in $\mathcal{N}_{\mathrm{x}}$ and all the viscous tensors $S_{a b}^{\mathrm{x}}, \mathcal{S}_{a b}^{\mathrm{xy}}$ and $s_{a b}^{\mathrm{xy}}$. The same result - that is, each $D_{a b}^{\mathrm{x}, \mathrm{e}}$ vanishes - holds even in a less rich situation when the model is built up with fewer viscous tensors. In that case we have to go back to eq. (112) and modify it accordingly. It is important to stress that we have shown that the full stress-energy-momentum tensor at equilibrium is made out of just the non-dissipative part, and that the dissipative parts of the total stress-energymomentum tensor do not contribute to the total energy density at second order.

However, we note that the energy minimum conditions in eq. (112) do not set the purely reactive terms to zero (eq. (30)). In fact, it only leads to

$$
\begin{aligned}
& \mathcal{M}_{\mathrm{n}} \frac{\partial \mathcal{N}_{\mathrm{n}}^{\mathrm{d}}}{\partial X_{\mathrm{n}}^{A}}=-\mathrm{R}_{A}^{\mathrm{sn}, \mathrm{e}} \\
& \mathcal{M}_{\mathrm{s}} \frac{\partial \mathcal{N}_{\mathrm{s}}^{\mathrm{d}}}{\partial X_{\mathrm{s}}^{A}}=-\mathrm{R}_{A}^{\mathrm{ns}, \mathrm{e}} .
\end{aligned}
$$

The reason for this is pretty clear as these terms do not enter the energy density formula. We nonetheless might want to consider the case where the equilibrium equations are exactly as the conservative ones. The motivation for this can be found in the derivation itself of the purely reactive terms. If the different species are comoving at the action level, there is no distinction between the different $X_{\mathrm{x}}^{A}$ and no resistive reactive term of this form would appear. We can enforce consistency with this observation in two ways: either we assume that we use the complete dependence on $X_{\mathrm{x}}^{A}$ in the conservative part, in which case

$$
\left.\frac{\partial \mathcal{N}_{\mathrm{x}}^{\mathrm{d}}}{\partial X_{\mathrm{x}}^{A}}\right|_{\mathrm{e}}=0 \Longrightarrow \mathrm{R}_{A}^{\mathrm{xy}, \mathrm{e}}=0
$$


or, we just set the terms $\overline{\mathrm{R}}_{a}^{\mathrm{x}}$ to zero, so that

$$
\left.\overline{\mathcal{M}}_{\mathrm{n}} \frac{\partial \mathcal{N}_{\mathrm{n}}^{\mathrm{d}}}{\partial X_{\mathrm{n}}^{A}}\right|_{\mathrm{e}}=\left.\overline{\mathcal{M}}_{\mathrm{s}} \frac{\partial \mathcal{N}_{\mathrm{s}}^{\mathrm{d}}}{\partial X_{\mathrm{s}}^{A}}\right|_{\mathrm{e}} .
$$

The latter, less restrictive assumption reminds us of the dynamical nature of chemical equilibrium in nature. Reactions happen also at equilibrium, although they do so in such a way that there is no net particle production. Such equilibrium reactions are key to explaining neutron star cooling.

Finally, it is quite easy to see that if we choose a different observer, such as the ones associated with the Eckart or Landau frame, the differences in the energy density will be of second order. Crucially, the equilibrium conditions in eq. (112) do not depend on the choice of frame.

\section{THE LAST PIECE OF THE PUZZLE}

In order to work out the perturbative expressions we need to expand the various dis-

sipative terms. It should now be clear that for the viscous stress tensors we can write ${ }^{24}$

$$
\begin{aligned}
\delta s_{A B}^{\mathrm{xy}} & =2 \frac{\partial \mathcal{N}_{\mathrm{x}}^{\mathrm{d}}}{\partial g_{\mathrm{y}}^{A B}} \delta \mathcal{M}_{\mathrm{x}}+2 \overline{\mathcal{M}}_{\mathrm{x}} \delta\left(\frac{\partial \mathcal{N}_{\mathrm{x}}^{\mathrm{d}}}{\partial g_{\mathrm{y}}^{A B}}\right), \\
\delta \mathcal{S}_{A B}^{\mathrm{xy}} & =2 \frac{\partial \mathcal{N}_{\mathrm{x}}^{\mathrm{d}}}{\partial g_{\mathrm{xy}}^{A B}} \delta \mathcal{M}_{\mathrm{x}}+2 \overline{\mathcal{M}}_{\mathrm{x}} \delta\left(\frac{\partial \mathcal{N}_{\mathrm{x}}^{\mathrm{d}}}{\partial g_{\mathrm{xy}}^{A B}}\right), \\
\delta S_{A B}^{\mathrm{x}} & =2 \frac{\partial \mathcal{N}_{\mathrm{x}}^{\mathrm{d}}}{\partial g_{\mathrm{x}}^{A B}} \delta \mathcal{M}_{\mathrm{x}}+2 \overline{\mathcal{M}}_{\mathrm{x}} \delta\left(\frac{\partial \mathcal{N}_{\mathrm{x}}^{\mathrm{d}}}{\partial g_{\mathrm{x}}^{A B}}\right)-\overline{\mathcal{M}}_{\mathrm{x}}\left(\delta \mathcal{N}_{\mathrm{x}}^{\mathrm{d}}\right) g_{A B}^{\mathrm{e}},
\end{aligned}
$$

where

$$
\begin{aligned}
& s_{A B}^{\mathrm{xy}}=2 \mathcal{M}_{\mathrm{x}} \frac{\partial \mathcal{N}_{\mathrm{x}}^{\mathrm{d}}}{\partial g_{\mathrm{y}}^{A B}}, \\
& \mathcal{S}_{A B}^{\mathrm{xy}}=2 \mathcal{M}_{\mathrm{x}} \frac{\partial \mathcal{N}_{\mathrm{x}}^{\mathrm{d}}}{\partial g_{\mathrm{xy}}^{A B}}, \\
& S_{A B}^{\mathrm{x}}=2\left(\mathcal{M}_{\mathrm{x}} \frac{\partial \mathcal{N}_{\mathrm{x}}^{\mathrm{d}}}{\partial g_{\mathrm{x}}^{A B}}-\frac{1}{2} \mathcal{M}^{\mathrm{x}} \mathcal{N}_{\mathrm{x}}^{\mathrm{d}} g_{A B}^{\mathrm{x}}\right) .
\end{aligned}
$$

${ }^{24}$ All the derivatives are intended to be evaluated at equilibrium. 
Similarly, for the "purely resistive" terms we have

$$
\delta \mathrm{R}_{A}^{\mathrm{xy}}=\frac{\partial \mathcal{N}_{\mathrm{x}}^{\mathrm{d}}}{\partial X_{\mathrm{y}}^{A}} \delta \mathcal{M}_{\mathrm{x}}+\overline{\mathcal{M}}_{\mathrm{x}} \delta\left(\frac{\partial \mathcal{N}_{\mathrm{x}}^{\mathrm{d}}}{\partial X_{\mathrm{y}}^{A}}\right)
$$

Since $\mathcal{N}_{\mathrm{x}}^{d}$ is a function of $\left(X_{\mathrm{x}}, X_{\mathrm{y}}, g_{\mathrm{x}}^{A B}, g_{\mathrm{y}}^{A B}, g_{\mathrm{xy}}^{A B}\right)$, its derivatives are as well, so that we have

$$
\begin{aligned}
\delta\left(\frac{\partial \mathcal{N}_{\mathrm{x}}^{\mathrm{d}}}{\partial X_{\mathrm{y}}^{A}}\right)= & \frac{\partial^{2} \mathcal{N}_{\mathrm{x}}^{\mathrm{d}}}{\partial X_{\mathrm{x}}^{B} \partial X_{\mathrm{y}}^{A}} \delta X_{\mathrm{x}}^{B}+\frac{\partial^{2} \mathcal{N}_{\mathrm{x}}^{\mathrm{d}}}{\partial X_{\mathrm{y}}^{B} \partial X_{\mathrm{y}}^{A}} \delta X_{\mathrm{y}}^{B}+\frac{\partial^{2} \mathcal{N}_{\mathrm{x}}^{\mathrm{d}}}{\partial g_{\mathrm{x}}^{B C} \partial X_{\mathrm{y}}^{A}} \delta g_{\mathrm{x}}^{B C} \\
& +\frac{\partial^{2} \mathcal{N}_{\mathrm{x}}^{\mathrm{d}}}{\partial g_{\mathrm{y}}^{B C} \partial X_{\mathrm{y}}^{A}} \delta g_{\mathrm{y}}^{B C}+\frac{\partial^{2} \mathcal{N}_{\mathrm{x}}^{\mathrm{d}}}{\partial g_{\mathrm{xy}}^{B C} \partial X_{\mathrm{y}}^{A}} \delta g_{\mathrm{xy}}^{B C}
\end{aligned}
$$

Similar results hold for the other variations that were not explicitly written in eq. (120) and eq. (122).

Concerning the purely reactive term we note that $u_{\mathrm{x}}^{a} \mathrm{R}_{a}^{\mathrm{yx}}=0$ automatically. Becuase we are doing an expansion with undetermined coefficients, we need to impose this by hand at every order; specifically, at the linear level. This then leads to

$$
\delta\left(u_{\mathrm{x}}^{a} \mathrm{R}_{a}^{\mathrm{yx}}\right)=\mathrm{R}_{A}^{\mathrm{yx}, \mathrm{e}}\left(w_{\mathrm{x}}^{A}-\dot{\xi}_{\mathrm{x}}^{A}\right)=0
$$

so that not only do we have $w_{\mathrm{x}}^{a}=\dot{\xi}_{\mathrm{x}}^{a}$ but also $w_{\mathrm{x}}^{A}=\dot{\xi}_{\mathrm{x}}^{A}$. This then means that we must have $u_{\mathrm{e}}^{a} \xi_{\mathrm{x}}^{b} X_{\mathrm{e} ; b a}^{D}=0$, which in turn implies that the orthogonality conditions for the viscous stress tensors

$$
S_{a b}^{\mathrm{x}} u_{\mathrm{x}}^{a}=\mathcal{S}_{a b}^{\mathrm{xy}} u_{\mathrm{x}}^{a}=s_{a b}^{\mathrm{xy}} u_{\mathrm{y}}^{a}=0
$$

are automatically satisfied at linear order.

From eq. (120) we can find the expansion for the spacetime viscous tensors through

$$
\begin{aligned}
& \delta S_{a b}^{\mathrm{x}}=\delta S_{D E}^{\mathrm{x}} \Psi_{\mathrm{e} a}^{D} \Psi_{\mathrm{e} b}^{E}-S_{D E}^{\mathrm{x}}\left(\xi_{\mathrm{x}, a}^{D} \Psi_{\mathrm{e} b}^{E}+\Psi_{\mathrm{e} a}^{D} \xi_{\mathrm{x}, b}^{E}\right) \\
& \delta s_{a b}^{\mathrm{xy}}=\delta s_{D E}^{\mathrm{xy}} \Psi_{\mathrm{e} a}^{D} \Psi_{\mathrm{e} b}^{E}-s_{D E}^{\mathrm{xy}}\left(\xi_{\mathrm{y}, a}^{D} \Psi_{\mathrm{e} b}^{E}+\Psi_{\mathrm{e} a}^{D} \xi_{\mathrm{y}, b}^{E}\right), \\
& \delta \mathcal{S}_{a b}^{\mathrm{xy}}=\delta \mathcal{S}_{D E}^{\mathrm{xy}} \Psi_{\mathrm{e} a}^{D} \Psi_{\mathrm{e} b}^{E}-\mathcal{S}_{D E}^{\mathrm{xy}}\left(\xi_{\mathrm{x}, a}^{D} \Psi_{\mathrm{e} b}^{E}+\Psi_{\mathrm{e} a}^{D} \xi_{\mathrm{y}, b}^{E}\right),
\end{aligned}
$$

while for the reactive terms associated with $s_{a b}^{\mathrm{xy}}$ and $\mathcal{S}_{a b}^{\mathrm{xy}}$ we have

$$
\begin{aligned}
\delta r_{a}^{\mathrm{xy}} & =\frac{1}{2} \delta s_{D E}^{\mathrm{xy}} \nabla_{a} g_{\mathrm{e}}^{D E}-\frac{1}{2} s_{D E}^{\mathrm{xy}} \partial_{a}\left[g^{b c}\left(\xi_{\mathrm{y}, b}^{D} \Psi_{\mathrm{e} c}^{E}+\Psi_{\mathrm{e} b}^{D} \xi_{\mathrm{y}, c}^{E}\right)\right] \\
\delta \mathcal{R}_{a}^{\mathrm{xy}} & =\frac{1}{4} \delta \mathcal{S}_{D E}^{\mathrm{xy}} \nabla_{a} g_{\mathrm{e}}^{D E}-\frac{1}{2} \mathcal{S}_{D E}^{\mathrm{xy}} g^{b c}\left(\xi_{\mathrm{x}, b}^{D} \nabla_{a} \Psi_{\mathrm{e} c}^{E}+\Psi_{\mathrm{e} b}^{D} \nabla_{a} \xi_{\mathrm{y}, c}^{E}\right)
\end{aligned}
$$


where we made use of the fact that $\left[\delta, \nabla_{a}\right]=0$ because of $\delta g^{a b}=0$ (see the discussed at the end of section IV).

Having "understood" how we may perturb the terms $R_{a}^{\mathrm{x}}$ and $D_{a b}^{\mathrm{x}}$, let us focus on the remaining pieces of the equation of motion. A quick look back at eq. (14) reveals that the only terms we still have to discuss are $\delta \Gamma_{\mathrm{x}}$ and $\delta \mu_{a}^{\mathrm{x}}$. For the particle creation rate we have (see eq. (99))

$$
\delta \Gamma_{\mathrm{x}}=\nabla_{a} \delta n_{\mathrm{x}}^{a}=\delta \dot{n}_{\mathrm{x}}+\nabla_{a}\left(\bar{n}_{\mathrm{x}} w_{\mathrm{x}}^{a}\right)
$$

while for the x-species momentum, we get

$$
\delta \mu_{a}^{\mathrm{x}}=\delta\left(\mathcal{B}_{\mathrm{x}} n_{\mathrm{x}}\right) u_{b}^{\mathrm{e}}+\overline{\mathcal{B}}_{\mathrm{x}} \bar{n}_{\mathrm{x}} w_{b}^{\mathrm{x}}+\sum_{\mathrm{y} \neq \mathrm{x}} \delta\left(\mathcal{A}_{\mathrm{xy}} n_{\mathrm{y}}\right) u_{b}^{\mathrm{e}}+\overline{\mathcal{A}}_{\mathrm{xy}} \bar{n}_{\mathrm{y}} w_{b}^{\mathrm{y}}
$$

Using the fact that we identified $\mathcal{M}_{\mathrm{x}}$ with $\mu_{\mathrm{x}}$ we have

$$
\begin{aligned}
\delta \mathcal{M}_{\mathrm{x}} & =\delta\left(-\mu_{\mathrm{x}}^{a} u_{a}^{\mathrm{x}}\right)=-\left(\bar{\mu}_{\mathrm{x}}^{a} w_{\mathrm{x}}^{a}+\delta \mu_{\mathrm{x}}^{a} u_{a}^{\mathrm{e}}\right) \\
& =\delta\left(\mathcal{B}_{\mathrm{x}} n_{\mathrm{x}}+\sum_{\mathrm{y} \neq \mathrm{x}} \mathcal{A}_{\mathrm{xy}} n_{\mathrm{y}}\right)
\end{aligned}
$$

and since $\mathcal{B}_{\mathrm{x}}$ and $\mathcal{A}_{\mathrm{xy}}$ are ultimately functions of $n_{\mathrm{x}}^{2}$ and $n_{\mathrm{xy}}^{2}$, we may use

$$
\begin{aligned}
& \delta \mathcal{B}_{\mathrm{x}}=\left(2 n_{\mathrm{x}} \frac{\partial \mathcal{B}_{\mathrm{x}}}{\partial n_{\mathrm{x}}^{2}}+n_{\mathrm{y}} \frac{\partial \mathcal{B}_{\mathrm{x}}}{\partial n_{\mathrm{xy}}^{2}}\right) \delta n_{\mathrm{x}}+\left(2 n_{\mathrm{y}} \frac{\partial \mathcal{B}_{\mathrm{x}}}{\partial n_{\mathrm{y}}^{2}}+n_{\mathrm{x}} \frac{\partial \mathcal{B}_{\mathrm{x}}}{\partial n_{\mathrm{xy}}^{2}}\right) \delta n_{\mathrm{y}}, \\
& \delta \mathcal{A}_{\mathrm{xy}}=\left(2 n_{\mathrm{x}} \frac{\partial \mathcal{A}_{\mathrm{xy}}}{\partial n_{\mathrm{x}}^{2}}+n_{\mathrm{y}} \frac{\partial \mathcal{A}_{\mathrm{xy}}}{\partial n_{\mathrm{xy}}^{2}}\right) \delta n_{\mathrm{x}}+\left(2 n_{\mathrm{y}} \frac{\partial \mathcal{A}_{\mathrm{xy}}}{\partial n_{\mathrm{y}}^{2}}+n_{\mathrm{x}} \frac{\partial \mathcal{A}_{\mathrm{xy}}}{\partial n_{\mathrm{xy}}^{2}}\right) \delta n_{\mathrm{y}},
\end{aligned}
$$

in eq. (130). This way, making use of definitions in eq. (104), we arrive at

$$
\delta \mathcal{M}_{\mathrm{x}}=\left(\overline{\mathcal{B}}_{\mathrm{x}} c_{\mathrm{x}}^{2}-\overline{\mathcal{A}}_{u u}^{\mathrm{xx}}\right) \delta n_{\mathrm{x}}-\left(\overline{\mathcal{A}}_{u u}^{\mathrm{ns}}+\bar{\chi}_{u u}^{\mathrm{ns}}\right) \delta n_{\mathrm{y}}
$$

and we see that the parameters that enter the dissipative fluid equations are the entrainment coefficients (and first derivatives; that is, second order derivatives of $\Lambda\left(n_{\mathrm{x}}^{2}, n_{\mathrm{xy}}^{2}\right)$ ) and the (up to second order) derivatives of the function $\mathcal{N}_{\mathrm{x}}\left(X_{\mathrm{x}}, X_{\mathrm{y}}, g_{\mathrm{x}}^{A B}, g_{\mathrm{y}}^{A B}, g_{\mathrm{xy}}^{A B}\right)$.

Having outlined the perturbative framework, it is natural to ask how many dissipative channels does the (general) model contain. Or, to be more specific; how many "dissipation coefficients" would have to be determined from microphysics. According to the expansion scheme we have developed so far, the perturbative expressions for the dissipative terms will ultimately involve all the second and first order derivatives of the $\mathcal{N}_{\mathrm{x}}^{d}$ when considered as 
functions of $X_{\mathrm{x}}, X_{\mathrm{y}}, g_{\mathrm{x}}^{A B}, g_{\mathrm{y}}^{A B}, g_{\mathrm{xy}}^{A B}$. Also, to make use of the model we need to specify the entrainment coefficients and their derivatives in the combinations from eq. (104).

The coefficients should be, in general, known once a specific model is chosen; that is, the explicit functional forms of $\Lambda$ and the $\mathcal{N}_{\mathrm{x}}^{d}$ have been determined. For example, if nuclear

physics calculations are used to determine these explicit forms, they must be done in such a way that the constraints which arise from requiring a meaningful equilibrium configuration are taken into account, and they must ensure that the Second Law of Thermodynamics is obeyed. If Onsager-type reasoning [10] is invoked to ensure $\Gamma_{\mathrm{s}}$ is positive (up to second order), then explicit use of

$$
T \Gamma_{\mathrm{s}}=-D_{b a}^{\mathrm{s}} \nabla^{b} u_{\mathrm{s}}^{a}-u_{\mathrm{s}}^{a} \mathrm{R}_{a}^{\mathrm{s}},
$$

where $T=-u_{\mathrm{s}}^{a} \mu_{a}^{\mathrm{s}}$ is the temperature, would have to be made.

\section{MODEL COMPARISON}

As an intuitive application of the formulation it is useful to make contact with existing models for general relativistic dissipative fluids, in particular, the classic work of LandauLifschitz and Eckart and the second-order Müller-Israel-Stewart model. Specifically, we want to understand how standard quantities (like shear and bulk viscosity) enter the present formalism. Therefore, we need to see if the dissipative terms of the existing models, $\left(q^{a}, \chi, \chi_{a b}\right)$, can be matched with terms in the action-based description. This procedure is fairly straightforward.

The action-based model provides the total fluid stress-energy-momentum tensor, so we only have to decompose it in the usual way:

$$
T^{a b}=(\bar{p}+\chi) \perp^{a b}+\varepsilon u^{a} u^{b}+2 q^{(a} u^{b)}+\chi^{a b} .
$$

In this expression, the fluxes are defined with respect to some observer with four-velocity $u^{a}$. In order to be consistent with the perturbative expansion outlined above, we take this observer to be associated with the thermodynamical equilibrium, i.e. $u^{a}=u_{\mathrm{e}}^{a}$. 


\section{A. Equating the Flux Currents}

Let us first consider the heat. We can read off the heat flux from the total stress-energymomentum tensor as

$$
q^{a}=-\varepsilon u_{\mathrm{e}}^{a}-T^{a b} u_{b}^{\mathrm{e}}=-\perp_{b}^{a} T^{b c} u_{c}^{\mathrm{e}}
$$

First, let us note there is no contribution coming from the dissipative part of the stressenergy-momentum tensor $D^{a b}$. In fact, making use of eqs. (114), (115) and (126), it is easy to show that $D^{a b} u_{a}^{\mathrm{e}}=\left(\delta D^{a b}\right) u_{a}^{\mathrm{e}}=\mathcal{O}(2)$. Let us therefore consider the non-dissipative part of $T^{a b}$. For the generalized pressure we have to first order

$$
\Psi=\Lambda+\sum_{x} n_{\mathrm{x}} \mu_{\mathrm{x}}=-\bar{\varepsilon}_{\mathrm{e}}+\bar{\mu} \bar{n}+\bar{T} \bar{s}+\sum_{x=n, s} \bar{n}_{\mathrm{x}} \delta \mu_{\mathrm{x}}=\bar{p}+\sum_{x=n, s} \bar{n}_{\mathrm{x}} \delta \mu_{\mathrm{x}}
$$

where we have used the minimum energy condition (eq. (108)) and the equilibrium Euler relation. Using

$$
\sum_{\mathrm{x}} n_{\mathrm{x}}^{a} \hat{\mu}_{\mathrm{x}}=\sum_{\mathrm{x}} n_{\mathrm{x}}^{a} \mu_{\mathrm{x}}+\mathcal{O}(2)=\sum_{\mathrm{x}}\left[\bar{n}_{\mathrm{x}} \bar{\mu}_{\mathrm{x}} u_{\mathrm{e}}^{a}+\bar{n}_{\mathrm{x}} \delta \mu_{\mathrm{x}} u_{\mathrm{e}}^{a}+\bar{\mu}_{\mathrm{x}}\left(\delta n_{\mathrm{x}} u_{\mathrm{e}}^{a}+\bar{n}_{\mathrm{x}} w_{\mathrm{x}}^{a}\right)\right]
$$

we then identify the heat flux as

$$
q^{a}=\sum_{\mathrm{x}} \bar{\mu}_{\mathrm{x}} \delta n_{\mathrm{x}}^{a}=\bar{\mu} \bar{n} w_{\mathrm{n}}^{a}+\bar{T} \bar{s} w_{\mathrm{s}}^{a}
$$

Here again we have repeatedly used the Euler relation and the minimum energy condition. We note that this quantity is consistent with the definition used in the classic models, see [37].

Let us now move on to the other fluxes and, as before, first focus on the non-dissipative contribution. It is easy to check that

$$
\begin{aligned}
T_{\text {n.d. }}^{a b}= & \left(\bar{p}+\sum_{\mathrm{x}} \bar{n}_{\mathrm{x}} \delta \mu_{\mathrm{x}}\right) g^{a b}+\left(\bar{p}+\bar{\varepsilon}_{\mathrm{e}}\right) u_{\mathrm{e}}^{a} u_{\mathrm{e}}^{b} \\
& +\sum_{\mathrm{x}}\left[\bar{\mu}_{\mathrm{x}} \bar{n}_{\mathrm{x}} u_{\mathrm{e}}^{b} w_{\mathrm{x}}^{a}+\bar{n}_{\mathrm{x}} u_{\mathrm{e}}^{a}\left(\delta \mu_{\mathrm{x}} u_{\mathrm{e}}^{b}+\mathcal{B}_{\mathrm{x}} \bar{n}_{\mathrm{x}} w_{\mathrm{x}}^{b}+\sum_{\mathrm{y} \neq \mathrm{x}} \mathcal{A}_{\mathrm{xy}} \bar{n}_{\mathrm{y}} w_{\mathrm{y}}^{b}\right)\right],
\end{aligned}
$$

so that, using the standard decomposition above one arrives at

$$
(\bar{p}+\chi) \perp^{a b}+\chi^{a b}=\perp_{c}^{a} \perp_{d}^{b} T^{c d}=T^{a b}+T^{a d} u_{d}^{\mathrm{e}} u_{\mathrm{e}}^{b}+T^{c b} u_{c}^{\mathrm{e}} u_{\mathrm{e}}^{a}+\varepsilon u_{\mathrm{e}}^{a} u_{\mathrm{e}}^{b} .
$$

If we now use the non-dissipative contribution $T_{\text {n.d. }}^{a b}$ in this equation, we get

$$
(\bar{p}+\chi) \perp^{a b}+\chi^{a b}=\left(\bar{p}+\sum_{\mathrm{x}} \bar{n}_{\mathrm{x}} \delta \mu_{\mathrm{x}}\right) \perp^{a b}=(\bar{p}+\delta \Psi) \perp^{a b}
$$


That is, there may be a first-order correction in the pressure coming from $T_{\text {n.d. }}^{a b}$ Let us consider the contribution due to the non-dissipative part. From eq. (126) we readily see that

$$
\perp_{c}^{a} \perp_{d}^{b} D^{c d}=D^{a b}=\delta D^{a b}
$$

Putting everything together, we have identified

$$
\begin{aligned}
\hat{\chi} & =\delta \Psi+\frac{1}{3} g_{a b} \delta D^{a b}, \\
\hat{\chi}^{a b} & =\delta D^{\langle a b\rangle}, \\
\hat{q}^{a} & =\bar{\mu} \bar{n} w_{\mathrm{n}}^{a}+\bar{T} \bar{s} w_{\mathrm{s}}^{a},
\end{aligned}
$$

where, as usual, the angle brackets mean that we are taking the trace-free symmetric part of the tensor. Also, we reintroduced the "hat" to stress that these fluxes are measured by the equilibrium observer.

\section{B. Example: A single viscous fluid}

We now consider the example of a two-species, single viscous fluid. The two species are matter, with non-equilibrium flux $n^{a}=n u_{\mathrm{f}}^{a}$, and entropy, with non-equilibrium flux $s^{a}=s u_{\mathrm{f}}^{a}$. In this simple case, we assume that the non-equilibrium fluxes remain parallel, meaning $w_{\mathrm{n}}^{a}=w_{\mathrm{s}}^{a}=w^{a}$ and therefore

$$
\begin{gathered}
n_{\mathrm{n}}^{a}=n u_{\mathrm{f}}^{a}=n\left(u_{\mathrm{e}}^{a}+w^{a}\right), \\
n_{\mathrm{s}}^{a}=s u_{\mathrm{f}}^{a}=s\left(u_{\mathrm{e}}^{a}+w^{a}\right),
\end{gathered}
$$

where again $u_{\mathrm{e}}^{a}$ is the equilibrium flow. In this case we do not have reactive terms because the two fluids are locked together from the beginning. Dissipation enters by assuming both currents depend on the (single) projected metric

$$
\begin{gathered}
\mathcal{N}_{\mathrm{n}}=\mathcal{N}_{\mathrm{n}}\left(X^{A}, g^{A B}\right), \\
\mathcal{N}_{\mathrm{s}}=\mathcal{N}_{\mathrm{s}}\left(X^{A}, g^{A B}\right) .
\end{gathered}
$$

In the equation of motion we will have additional terms due to $S_{a b}^{\mathrm{s}}$ and $S_{a b}^{\mathrm{n}}$.

Because there is only one matter species, the creation rate $\Gamma_{\mathrm{n}}$ has to vanish; this implies

$$
\Gamma_{\mathrm{n}}=-\frac{1}{\mu_{\mathrm{n}}} S_{a b}^{\mathrm{n}} \nabla^{a} u_{\mathrm{f}}^{b}=0 \Longrightarrow S_{a b}^{\mathrm{n}}=0
$$


As a result, the final form of the non-linear equation of motion is

$$
2 n_{\mathrm{n}}^{a} \nabla_{[a} \mu_{b]}^{\mathrm{n}}+2 n_{\mathrm{s}}^{a} \nabla_{[a} \mu_{b]}^{\mathrm{s}}+\Gamma_{\mathrm{s}} \mu_{b}^{\mathrm{s}}=-\nabla^{a} S_{a b}^{\mathrm{s}}
$$

Note that, when we linearize, the term involving $\Gamma_{\mathrm{s}}$ will not appear in the equations, because $\Gamma_{\mathrm{s}}$ has no linear term - entropy is expanded around a maximum-leaving only the secondorder term, which is positive-definite so that the Second Law of Thermodynamics can be satisfied.

Our next step is to use the expansion formalism developed in the previous sections to determine the explicit form of the viscous stress tensor $S_{a b}^{\mathrm{s}}$. Let us start by considering the equilibrium (minimum energy) conditions. Clearly, we should have

$$
S_{A B}^{\mathrm{s}, \mathrm{e}}=2 \mathcal{M}_{\mathrm{s}} \frac{\partial \mathcal{N}_{\mathrm{s}}^{\mathrm{d}}}{\partial g^{A B}}=0 \Longrightarrow \frac{\partial \mathcal{N}_{\mathrm{s}}^{\mathrm{d}}}{\partial g^{A B}}=0
$$

It also makes sense to assume $\partial \mathcal{N}_{\mathrm{s}}^{d} /\left.\partial X^{A}\right|_{\mathrm{e}}=0$. To see why, let us forget for the moment that the two species are locked together and consider the purely reactive term:

$$
\begin{aligned}
\bar{R}_{A}^{\mathrm{s}} & =\mathcal{M}_{\mathrm{n}} \frac{\partial \mathcal{N}_{\mathrm{n}}^{\mathrm{d}}}{\partial X_{\mathrm{s}}^{A}}-\mathcal{M}_{\mathrm{s}} \frac{\partial \mathcal{N}_{\mathrm{s}}^{\mathrm{d}}}{\partial X_{\mathrm{n}}^{A}}=-\mathcal{M}_{\mathrm{s}} \frac{\partial \mathcal{N}_{\mathrm{s}}^{\mathrm{d}}}{\partial X_{\mathrm{s}}^{A}}-\mathcal{M}_{\mathrm{s}} \frac{\partial \mathcal{N}_{\mathrm{s}}^{\mathrm{d}}}{\partial X_{\mathrm{n}}^{A}} \\
& =-2 \mathcal{M}_{\mathrm{s}} \frac{\partial \mathcal{N}_{\mathrm{s}}^{\mathrm{d}}}{\partial X_{\mathrm{s}}^{A}}=-2 \mathcal{M}_{\mathrm{s}} \frac{\partial \mathcal{N}_{\mathrm{s}}^{\mathrm{d}}}{\partial X^{A}}=0,
\end{aligned}
$$

where we initially distinguished between the two constituents' matter-space coordinates, and used the equilibrium condition. The condition $\partial \mathcal{N}_{\mathrm{x}}^{\mathrm{d}} / \partial X^{A}=0$ is motivated by the fact that the reactive term vanishes (because the two currents are effectively locked).

As a result of these constraints we have $\delta \mathcal{N}_{\mathrm{x}}^{\mathrm{d}}=\mathcal{O}(2), \delta \Psi=\mathcal{O}(2)$ and the viscous stress tensor becomes (see eqs. (120) and (126))

$$
\begin{aligned}
\delta S_{a b}^{\mathrm{s}} & =\left[2 \overline{\mathcal{M}}_{\mathrm{s}} \delta\left(\frac{\partial \mathcal{N}_{\mathrm{s}}^{\mathrm{d}}}{\partial g^{A B}}\right)\right] \Psi_{\mathrm{e} a}^{A} \Psi_{\mathrm{e} b}^{B} \\
& =-2 \bar{T}\left[\frac{\partial \mathcal{N}_{\mathrm{s}}^{\mathrm{d}}}{\partial X^{C} \partial g^{A B}} w^{C}+2 \frac{\partial \mathcal{N}_{\mathrm{s}}^{\mathrm{d}}}{\partial g^{D E} \partial g^{A B}} D^{(D} w^{E)}\right] \Psi_{\mathrm{e} a}^{A} \Psi_{\mathrm{e} b}^{B},
\end{aligned}
$$

where we have used the linearization procedure outlined in appendix A which shows

$$
\delta X^{A} \sim-w^{A}, \quad \delta g^{A B} \sim-2 D^{(A} w^{B)} .
$$

Given this, we can write the entropy production rate as (see eq. (133))

$$
\Gamma_{\mathrm{s}}=+\left[A_{C A B} w^{C}+\Sigma_{D E A B} D^{(D} w^{E)}\right]\left(\sigma^{A B}+\frac{1}{3} \theta g^{A B}\right) \geq 0,
$$


where we have introduced the two tensors:

$$
\begin{aligned}
A_{C A B} & =2 \frac{\partial \mathcal{N}_{\mathrm{s}}^{d}}{\partial X^{C} \partial g^{A B}}, \\
\Sigma_{D E A B} & =4 \frac{\partial \mathcal{N}_{\mathrm{s}}^{d}}{\partial g^{D E} \partial g^{A B}} .
\end{aligned}
$$

Noting that $D^{(D} w^{E)}=\sigma^{D E}+\frac{1}{3} \theta g^{D E}$, we can clearly satisfy the second law by assuming ${ }^{25}$

$$
\begin{aligned}
A_{C A B} & =0 \\
\Sigma_{D E A B} & =\frac{\zeta}{\bar{T}} g_{A B}^{\mathrm{e}} g_{D E}^{\mathrm{e}}+2 \frac{\eta}{\bar{T}}\left[g_{D(A}^{\mathrm{e}} g_{E) B}^{\mathrm{e}}-\frac{2}{3} g_{A B}^{\mathrm{e}} g_{D E}^{\mathrm{e}}\right]
\end{aligned}
$$

with $\zeta, \eta \geq 0$ so that

$$
\Gamma_{s}=\frac{\zeta}{\bar{T}} \theta_{\mathrm{e}}^{2}+\frac{\eta}{\bar{T}} \sigma_{\mathrm{e}}^{a b} \sigma_{a b}^{\mathrm{e}} \geq 0
$$

It also follows that, the only viscosity tensor of the model is

$$
S_{a b}^{\mathrm{s}}=\chi_{a b}+\chi \perp_{a b}=\frac{1}{3} \zeta \theta_{\mathrm{e}} \perp_{a b}+\eta \sigma_{a b}^{\mathrm{e}} .
$$

With these relations we have recovered the usual relativistic Navier-Stokes equations (the Landau-Lifschitz-Eckart model for a viscous fluid).

Let us point out that, to write down the full set of equations one should also expand the "Euler part" of the equation of motion, i.e. the left-hand-side of eq. (147). We have provided all the ingredients necessary for the explicit calculation, but leave it out as it is not new and not particularly relevant for the present discussion (see also [37] for further details).

\section{CAUSALITY AND TELEGRAPH-TYPE EQUATIONS}

As a practical example of the first-order expansion we produced the model for a single (bulk and shear) viscous fluid, and showed how this leads to the expected form of the relativistic Navier-Stokes equations. The derivation shows the action-based formalism contains within it the previous models. It also clear that the formalism developed allows us to consider much more complicated settings, should we need to do so. However, the discussion of the

\footnotetext{
25 Note that within this model it is easy to account for a non-isotropic response in the velocity-gradients to viscosity relation.
} 
first-order results is clearly not complete, because the final set of equations is widely known to suffer from causality/stability issues. In fact, the work of Hiscock and Lindblom [42] has shown these first-order models to be generically unstable. In practice, this means that if we set the system to deviate slightly from an equilibrium state, the deviations will grow exponentially and eventually diverge. Conversely, the second-order Müeller-Israel-Stewart theories have been shown to satisfy the conditions for stability and causality [see 8]. This has led to the common belief that all possible first-order theories are unstable and acausal, and that for these issues to be solved one has to go to second order.

The issue has recently drawn further attention because of the relevance of (general relativistic) dissipative fluid models in the new gravitational-wave era and for the modelling of heavy-ion collisions. In particular, in recently proposed field-theory-based models (see $[17,18])$ one postulates that the thermodynamic fluxes can be expanded in terms of the usual hydrodynamic variables and their derivatives. Halting the derivative expansion at first-order and performing a stability analysis, the authors showed that there exist a consistent set of constraints on the expansion parameters such that these models pass some of the stability and causality conditions.

A recent analysis by Gavassino et al. [43] shed new light on this matter, showing that Landau-Lifschitz-Eckart model instabilities are due to the enforcement of the Second Law on an entropy function that is not maximized at equilibrium, while the field-theory-based models can be made stable by allowing for small violation of the Second Law. Neither of these are, of course, "true" representations of the anticipated physics.

Another important aspect of the problem is provided by [39, 40], where it is demonstrated that, for a fluid model with heat-flux, one can resolve the stability/causality issues at first order by properly accounting for the entrainment between matter and entropy currentsretaining the compatibility with the Second Law. Because of this, it is reasonable to believe one can do the same at the first-order level also in different contexts. We will now discuss these issues using the single viscous fluid as a case study.

Essentially, the problem must be addressed in a different way, as the key ingredient used to solve the heat-flux case (see $[39,40]$ ) accounting for the entropy inertia, will not work for the present case as it does not involve relative flows. To make progress, we need to make a slight modification to the formalism. Specifically, we will sketch out how one can obtain - taking the action-based formalism as the starting point - a set of equations that is 
consistent with linearizing the equations of Müeller-Israel-Stewart. The argument is similar to that of Rational Thermodynamics, which stresses the importance of the "principle of memory or heredity" (see [38]).

The argument can be motivated with a Newtonian example, which highlights what needs to be changed in the formalism in order to enforce a causal behaviour. It makes intuitive sense that, if we want to implement causality in the model we need to account for some delay in the system's response. Following what is usually done in microphysical many-body theories, we can assume the response of the system to be non-local in time. If we consider a bulk-viscous fluid, this can be done by assuming that the trace part of the viscous stress tensor is related to the expansion rate in such a way that

$$
S=\int_{0}^{t} q\left(t-t^{\prime}\right) \theta\left(t^{\prime}\right) d t^{\prime}
$$

where the kernel $q$ is yet to be determined. Using a simple result that is valid for functions defined through integrals (functionals), namely

$$
\begin{aligned}
& \Phi(x)=\int_{\alpha(x)}^{\beta(x)} f(x, t) d t \\
& \frac{\partial \Phi}{\partial x}(x)=f(x, \beta(x)) \frac{\partial \beta}{\partial x}-f(x, \alpha(x)) \frac{\partial \alpha}{\partial x}+\int_{\alpha(x)}^{\beta(x)} \frac{\partial f}{\partial x}(x, t) d t
\end{aligned}
$$

we have

$$
\dot{S}=\partial_{t} S=q(0) \theta(t)+\int_{0}^{t} \partial_{t}\left[q\left(t-t^{\prime}\right)\right] \theta\left(t^{\prime}\right) d t^{\prime} .
$$

We can get a Telegraph-type equation for the flux by assuming

$$
q\left(t-t^{\prime}\right)=-\frac{\zeta}{\tau} e^{-\left(t-t^{\prime}\right) / \tau}
$$

In fact, this would lead to

$$
S+\tau \dot{S}=-\zeta \theta
$$

where we assumed that the response function kernel satisfies $q(0)=-\zeta / \tau$. In effect, the instantaneous response is the "Navier-Stokes" behaviour, while $\tau$ represents the typical timescale over which the system retains a memory of the past.

This simple example tells us two things. First, we can get an equation for the fluxes by assuming the kernel $q$ to be as above. Second, a delay in the system response can be implemented by assuming the flux to be a functional (not just a function) of the corresponding generalized force, in this case the expansion rate. 
Suppose we try to implement this lesson in the action-based formalism. This will be quite straightforward, subject to the following caveat: The (functional) integration should extend (at most) to the whole spacetime region causally connected with each point. In the present example, we will assume that the analysis is done locally in space but not necessarily in time, such as the world-tube formed by the spatial part of the region $\delta \mathcal{M}$ in fig. 1 . Even though there are no general proofs to this effect, we are assuming that instilling causality and stability in the world-tubes that can be used to fill out a system is probably a necessary condition for causality and stability to apply globally.

The next step is to assume that the normalization $\mathcal{N}_{\mathrm{s}}^{d}$ is a functional of $g^{A B}$ instead of a function. This means that we can write

$$
\begin{aligned}
\mathcal{N}_{\mathrm{x}}^{d}\left[g^{A B}\right](x) & =\mathcal{N}_{\mathrm{x}}^{d}\left[g_{\mathrm{e}}^{A B}\right](x)+\int \frac{\delta \mathcal{N}_{\mathrm{x}}^{d}}{\delta g^{A B}}\left(x, x^{\prime}\right) \delta g^{A B}\left(x^{\prime}\right) d^{4} x^{\prime} \\
& +\int \frac{\delta^{2} \mathcal{N}_{\mathrm{x}}^{d}}{\delta g^{A B} \delta g_{C D}}\left(x, x^{\prime}\right) \delta g^{A B}\left(x^{\prime}\right) \delta g^{C D}\left(x^{\prime}\right) d^{4} x^{\prime}
\end{aligned}
$$

where the first two terms vanish because (i) $\mathcal{N}_{\mathrm{x}}^{d}$ vanishes at equilibrium, and (ii) the minimum energy condition. Again, we assume that (the generalized version of) $A_{A B C}$ of eq. (152) is zero.

The key step is to replace the ordinary partial derivatives with functional derivatives in the various expressions we have discussed, so that the viscous stress tensor will be (see eq. (150))

$$
S_{A B}=2 \bar{T} \delta\left(\frac{\delta \mathcal{N}_{\mathrm{x}}^{d}}{\delta g^{A B}}\right)=2 \bar{T} \int \frac{\delta^{2} \mathcal{N}_{\mathrm{x}}^{d}}{\delta g^{A B} \delta g^{C D}} \delta g^{C D}\left(x^{\prime}\right) d^{4} x^{\prime}
$$

Formally we can introduce a set of spatial coordinates $\bar{x}$ comoving with the equilibrium observer attached to the world-tube, and take the time coordinate to be the equilibrium worldline's proper time $\tau$. Also, to enforce locality in space, and a retarded response in time, we may use

$$
\frac{\delta^{2} \mathcal{N}_{\mathrm{x}}^{d}}{\delta g^{A B} \delta g^{C D}}=\frac{1}{4} \Sigma_{A B C D}\left(\bar{x}, \tau-\tau^{\prime}\right) \delta^{3}\left(\bar{x}-\bar{x}^{\prime}\right) \Theta\left(\tau-\tau^{\prime}\right)
$$

We can also assume the fluid viscous response to be isotropic (as before) and set

$$
\Sigma_{A B C D}=\Sigma_{A B C D}^{\mathrm{b}}+\Sigma_{A B C D}^{\mathrm{s}}
$$


where

$$
\begin{aligned}
\Sigma_{A B C D}^{\mathrm{b}} & =\frac{\zeta(x)}{\bar{T}} g_{A B}^{\mathrm{e}} g_{C D}^{\mathrm{e}} q_{\mathrm{b}}\left(\tau-\tau^{\prime}\right) \delta^{3}\left(\bar{x}-\bar{x}^{\prime}\right) \\
\Sigma_{A B C D}^{\mathrm{s}} & =2 \frac{\eta(x)}{\bar{T}}\left(g_{A(C}^{\mathrm{e}} g_{B) D}^{\mathrm{e}}-\frac{2}{3} g_{A B}^{\mathrm{e}} g_{C D}^{\mathrm{e}}\right) q_{\mathrm{s}}\left(\tau-\tau^{\prime}\right) \delta^{3}\left(\bar{x}-\bar{x}^{\prime}\right)
\end{aligned}
$$

We retain the structure and symmetries from before, but introduce two different convolutions $q_{\mathrm{b}}$ and $q_{\mathrm{s}}$ to account for different response to bulk and shear strain rates. Clearly, we recover the Navier-Stokes limit if we assume there is no delay in the response functions, i.e. let $q_{\mathrm{b}, \mathrm{s}}\left(\tau-\tau^{\prime}\right)=\delta\left(\tau-\tau^{\prime}\right)$. We can make also the fluxes satisfy an equation of the Telegraph-type by choosing the response function as

$$
\tau^{\prime} q_{\mathrm{b}}\left(\tau-\tau^{\prime}\right) \propto e^{-\left(\tau-\tau^{\prime}\right) / t_{\mathrm{b}}}
$$

and similar for $q_{\mathrm{s}}$, where we introduce two different timescales $t_{\mathrm{b}}$ and $t_{\mathrm{s}}$.

Let us focus on the bulk viscosity contribution. Intuitively, one would like to recover the Navier-Stokes response in the limit of very short timescales $t_{\mathrm{b}} \rightarrow 0$. It is clear from the previous expression that to do so, we need to let the bulk viscosity coefficient diverge in the short timescale limit. That is, we need

$$
\zeta(x) e^{-\left(\tau-\tau^{\prime}\right) / t_{\mathrm{b}}} \underset{t_{\mathrm{b}} \rightarrow 0}{\Longrightarrow} \zeta_{N S}
$$

where $\zeta_{N S}$ is the Navier-Stokes bulk viscosity coefficient. This is in accord with the parabolic limit of [44]. A similar result holds for the shear viscosity.

In essence, we have shown how we can implement a causal response in the action-based model by assuming that $S_{A B}$ (and therefore $D_{a b}$ as well) is an integral function of $g^{A B}$. The question then is, does this mean that the final fluid equations are integro-differential equations? Fortunately the answer is no. In fact, we have shown that, by a suitable choice of the response function $q\left(\tau-\tau^{\prime}\right)$, the fluxes satisfy an equation of the Telegraph-type. Therefore, instead of solving an integro-differential equation, one should treat $S_{a b}^{\mathrm{s}}=\chi \perp_{a b}$ $+\chi_{a b}$ as an unknown in eq. (147), and add to the system the following two equations

$$
\begin{aligned}
\chi+t_{\mathrm{b}} \dot{\chi} & =-\zeta \theta, \\
\chi_{a b}+t_{\mathrm{s}} \dot{\chi}_{a b} & =-\eta \sigma_{a b} .
\end{aligned}
$$

This means that, at the end of the day, to actually solve a set of differential dissipative equations at first order, we have to treat the fluxes as additional unknowns, for which one 
has to provide equations that are not given by the stress-energy-momentum conservation law $\nabla_{a} T^{a b}=0$. This is reminiscent of the EIT paradigm, where one postulates from the beginning an entropy function that depends on an additional set of quantities - the thermodynamic fluxes. The difference is in the fact that the microphysical origin of the equation for the fluxes is now clear. It is worth noting that equations of the Telegraph-type for the fluxes cannot be obtained in the field-theory-based models, as the constitutive equations are given in terms of the usual equilibrium variables (like $\mu, T$ ) and their derivatives - so that terms with derivatives of the fluxes (like $\dot{\chi}$ ) do not appear.

Equation (170) is (formally) the same as in the linearized version of Müeller-Israel-Stewart model, which has been shown to be stable and causal. In theory, nothing prevents us from choosing a different form for the retarded response $q$ which could lead to non-causal behaviour. However, the form of $q$ suggested above has a clear interpretation and is microphysically motivated. If one wants to come up with an alternative, this would need to be motivated by microphysical arguments, as well.

Finally, let us consider the constraints that follows from the Second Law. It makes sense to start by checking if and how the formula for the particle production rate is changed by the modifications introduced to enforce causality. We now have (see eq. (29))

$$
\begin{aligned}
\mu_{\mathrm{x}} \Gamma_{\mathrm{x}} & =\frac{1}{3 !} \mu_{\mathrm{x}}^{A B C} \frac{d n_{A B C}^{\mathrm{x}}}{d \tau_{\mathrm{x}}}=\overline{\mathcal{M}}_{\mathrm{x}} \frac{d}{d \tau_{\mathrm{x}}}\left(\overline{\mathcal{N}}_{\mathrm{x}}^{c}+\overline{\mathcal{N}}_{\mathrm{x}}^{d}\right) \\
& =\mathcal{M}_{\mathrm{x}}\left(\frac{d \mathcal{N}_{\mathrm{x}}^{d}}{d \tau_{\mathrm{x}}}+\frac{1}{2} \mathcal{N}_{\mathrm{x}}^{d} g_{A B}^{\mathrm{x}} \frac{d g_{\mathrm{x}}^{A B}}{d \tau_{\mathrm{x}}}\right)
\end{aligned}
$$

In the case of a single viscous fluid, since $\mathcal{N}_{\mathrm{x}}^{d}=\mathcal{O}(2)$ and $\dot{g}_{\mathrm{x}}^{A B}=\mathcal{O}(1)$, the result simplifies to

$$
\Gamma_{\mathrm{x}}=\frac{d \mathcal{N}_{\mathrm{x}}^{d}}{d \tau}+\mathcal{O}(3)
$$

If we focus on the entropy and use the fact that $\mathcal{N}_{\mathrm{s}}^{d}=\mathcal{N}_{\mathrm{s}}^{d}\left[g^{A B}\right]$ as above (see eq. (163)) then we have

$$
\begin{aligned}
\Gamma_{s} & =+\int \frac{\partial}{\partial \tau}\left(\frac{\delta^{2} \mathcal{N}_{\mathrm{s}}^{d}}{\delta g^{A B} \delta g^{C D}}\left(\bar{x}, \tau, \bar{x}^{\prime}, \tau^{\prime}\right)\right) \delta g^{A B}\left(\bar{x}^{\prime}, \tau^{\prime}\right) \delta g^{C D}\left(\bar{x}^{\prime}, \tau^{\prime}\right) d^{4} x^{\prime} \\
& =+\int \frac{\partial}{\partial \tau}\left(\Sigma_{A B C D}\left(\bar{x}, \tau, \bar{x}^{\prime}, \tau^{\prime}\right)\right)\left(\tau^{\prime}\right)^{2} D^{(A} w^{B)} D^{(C} w^{D)} d^{4} x^{\prime}
\end{aligned}
$$


As a result, the entropy production rate due to bulk- and shear viscosity becomes

$$
\begin{aligned}
\Gamma_{\mathrm{s}}^{\mathrm{bulk}} & =\int d \tau^{\prime} \frac{\tau \tau^{\prime}}{\tau_{\mathrm{b}}} \frac{\zeta}{\bar{T}} e^{-\left(\tau-\tau^{\prime}\right) / t_{\mathrm{b}}} \theta_{\mathrm{e}}^{2} \\
\Gamma_{\mathrm{s}}^{\text {shear }} & =\int d \tau^{\prime} \frac{\tau \tau^{\prime}}{\tau_{\mathrm{s}}} \frac{\eta}{\bar{T}} e^{-\left(\tau-\tau^{\prime}\right) / t_{\mathrm{s}}} \sigma_{\mathrm{e}}^{a b} \sigma_{a b}^{\mathrm{e}} .
\end{aligned}
$$

Assuming the bulk and shear channels to be independent, we can satisfy the Second Law by assuming the viscosity coefficients $\zeta, \eta$ (as well as the relaxation timescales $\tau_{\mathrm{b}}, \tau_{\mathrm{s}}$ ) to be positive. This is clearly consistent with the Navier-Stokes limit and, at the end of the day, the model should be complete at first order.

\section{CONCLUSIONS AND FINAL REMARKS}

We have considered the close-to-equilibrium regime of the action-based model of Andersson and Comer [15] for dissipative multi-fluid systems. In particular, we have shown that, starting from a set of fully non-linear dynamical equations with only the fluxes as the degrees of freedom, an expansion with respect to (a self-consistently defined) equilibrium can be introduced in a clear fashion, with the line of reasoning being similar to that of usual hydrodynamical perturbation theory.

After discussing the aspects of equilibrium which can be inferred from the action-based model itself, we established how to construct the expansion in deviations away from equilibrium in a general setting, so that the framework is of wider relevance. In the process we demonstrated the importance of the frame-of-reference of the equilibrium observer. We also noted that the construction promotes the role of the matter space: Instead of it being a mathematical "trick" to facilitate a constrained variation, it might well be the arena where the microphysical details are encoded in the general relativistic regime. This is a novel perspective that needs further discussion and consideration.

We then focused on a particular first-order viscous fluid model, with shear- and bulkviscosity, paying particular attention to the key causality issues. We showed that causal behaviour can be linked to a retarded response function that keeps track of a system's history. The specific form of the response function can be modelled in a phenomenological way - as we did - but should ideally be provided by specific microphysical calculations, for instance by means of the fluctuation-dissipation theorem (see [45] for a general discussion 
and [38] for comments on its role from the EIT perspective). ${ }^{26}$ In a sense, the action-based model provides the "context", determining the geometric structure and form of the equations of motion, while the detailed microphysics is encoded in the specific response function.

Building the first-order expansion we made this connection clear, and showed how and where the microphysics enters the discussion. We note that, in contrast with recent fieldtheory-based models, we paid attention to the compatibility with the Second Law of Thermodynamics. An interesting outcome of this analysis is that we showed-contrary to the Müeller-Israel-Stewart line of reasoning - that to implement a causal response in the model there is no need to go to second order in deviations from equilibrium. This has already been shown for the heat-flux problem (see [39, 40]), where the Cattaneo-type equation for the heat flux is ultimately related to the multi-fluid nature of the problem. The entrainment effect (through which the entropy current gains an effective mass [48]) results in an inertial heat response. The case of a single viscous fluid is different since its retarded response cannot be associated with the multifluid nature of the problem.

As the general model begins with a fully non-linear set of field equations the route to further extensions is - at least at the formal level-quite clear. A natural next step would be the modelling of a viscous fluid allowing for the heat to flow differently from the matter. This application should be fairly straightforward since the two main issues of the problem have now been studied separately. A more challenging step will be the inclusion of superfluids. The presence of currents that persist for very long times changes drastically the non-dissipative limit. The model would require the use of more than one equilibrium worldline congruence [15, 49], one for each "superfluid condensate" and one for all the remaining constituents. We plan to investigate these issues - and the connection to neutron star astrophysics - at a later date.

\section{ACKNOWLEDGMENTS}

NA acknowledges support from STFC via grant no. ST/R00045X/1.

${ }^{26}$ We also note that there have been recent efforts to make explicit use of the fluctuation-dissipation theorem to compute response coefficients through Green-Kubo-like formulae, see [46, 47]-although in a special relativistic setting. Nevertheless, there are similarities with the way we deal with causality issues here. 


\section{Appendix A: On the linearization procedure}

Here we consider in more detail the linearization introduced above in eq. (151), motivating it using the method of characteristics. This method is used to solve hyperbolic partial differential equations and is valid in particular for first order linear equations of the type we are dealing with.

We want to find a formal solution to

$$
\dot{\xi}_{\mathrm{x}}^{D}=u^{a} \partial_{a} \xi_{\mathrm{x}}^{D}=\frac{\partial}{\partial \tau} \xi^{D}(\bar{x}, \tau)=w_{\mathrm{x}}^{D}(\bar{x}, \tau)
$$

The idea is to solve the equation along its characteristic curves $(\bar{x}(s), \tau(s))$, in order to deal with an ordinary differential equation instead;

$$
\frac{d}{d s} \xi_{\mathrm{x}}^{D}(\bar{x}(s), \tau(s))=w_{\mathrm{x}}^{D}(\bar{x}(s), \tau(s))
$$

If we now use the chain rule

$$
\frac{d}{d s} \xi_{\mathrm{x}}^{D}=\frac{\partial \xi_{x}^{D}}{\partial \bar{x}} \frac{d \bar{x}}{d s}+\frac{\partial \xi_{x}^{D}}{\partial \tau} \frac{d \tau}{d s}
$$

and set

$$
\frac{d \bar{x}}{d s}=0, \quad \frac{d \tau}{d s}=1
$$

then we see that eq. (A2) is the same as eq. (A1). Solving the characteristic curves equation as $\tau(s)=s$ and $x(s)=x_{0}$, the formal solution to eq. (A1) is

$$
\xi_{\mathrm{x}}^{D}(\bar{x}, \tau)=\int_{\tau_{0}}^{\tau} w_{\mathrm{x}}^{D}\left(\bar{x}, \tau^{\prime}\right) d \tau^{\prime}
$$

The linearization approximation consists in writing $w_{\mathrm{x}}^{D}\left(\bar{x}, \tau^{\prime}\right) \approx w_{\mathrm{x}}^{D}(\bar{x}, \tau)+\ldots$ in the last integral, which means that we get

$$
\begin{aligned}
\delta X_{\mathrm{x}}^{A}(\bar{x}, \tau) & =-\xi_{\mathrm{x}}^{A}=-\left(\tau-\tau_{0}\right) w_{\mathrm{x}}^{A}(\bar{x}, \tau) \\
\delta g_{\mathrm{x}}^{A B}(\bar{x}, \tau) & =-2 D^{(A} \xi_{\mathrm{x}}^{B)}=-2\left(\tau-\tau_{0}\right) D^{(A} w_{\mathrm{x}}^{B)}(\bar{x}, \tau)
\end{aligned}
$$

[1] C. Eckart, Phys. Rev. 58, 919 (1940).

[2] L. D. Landau and E. M. Lifshitz, Fluid mechanics (Pergamon Press, 1959). 
[3] I. Muller, Z. Phys. 198, 329 (1967).

[4] W. Israel, Annals of Physics 100, 310 (1976).

[5] J. M. Stewart, Proceedings of the Royal Society of London Series A 357, 59 (1977).

[6] W. Israel and J. M. Stewart, Annals of Physics 118, 341 (1979).

[7] W. Israel and J. M. Stewart, Proceedings of the Royal Society of London Series A 365, 43 (1979).

[8] W. A. Hiscock and L. Lindblom, Annals of Physics 151, 466 (1983).

[9] W. A. Hiscock and L. Lindblom, Physics Letters A 131, 509 (1988).

[10] L. Onsager, Phys. Rev. 37, 405 (1931).

[11] N. Andersson and G. L. Comer, Classical and Quantum Gravity 23, 5505 (2006).

[12] B. Haskell, N. Andersson, and G. L. Comer, Phys. Rev. D 86, 063002 (2012).

[13] B. Carter, Proceedings of the Royal Society 433, 45 (1991).

[14] D. Priou, Phys. Rev. D 43, 1223 (1991).

[15] N. Andersson and G. L. Comer, Classical and Quantum Gravity 32, 075008 (2015).

[16] B. Carter, in Relativistic Fluid Dynamics, edited by A. M. Anile and Y. Choquet-Bruhat (Springer Berlin Heidelberg, Berlin, Heidelberg, 1989), pp. 1-64.

[17] P. Kovtun, Journal of High Energy Physics 2019, 34 (2019).

[18] F. S. Bemfica, M. M. Disconzi, and J. Noronha, Phys. Rev. D 100, 104020 (2019).

[19] G. L. Comer and R. Joynt, Phys. Rev. D 68, 023002 (2003).

[20] N. Chamel, J. Low Temp. Phys. 189, 328 (2017).

[21] B. F. Schutz and R. Sorkin, Annals of Physics 107, 1 (1977).

[22] R. Prix, Phys. Rev. D 69, 043001 (2004).

[23] B. Carter and H. Quintana, Proceedings of the Royal Society of London Series A 331, 57 (1972).

[24] S. Carroll, Spacetime and Geometry (Cambridge University Press, 2019).

[25] J. L. Friedman and B. F. Schutz, Astrophys. J. 221, 937 (1978).

[26] M. Karlovini and L. Samuelsson, Classical and Quantum Gravity 24, 3171 (2007).

[27] G. L. Comer and D. Langlois, Class. Quant. Grav. 10, 2317 (1993).

[28] R. C. Tolman, Science 77, 291 (1933).

[29] R. C. Tolman, Science 77, 313 (1933).

[30] M. Fukuma and Y. Sakatani, Phys. Rev. E 84, 026315 (2011). 
[31] E. Gourgoulhon, in CompStar 2010: School and Workshop on Computational Tools for Compact Star Astrophysics (2010).

[32] F. Becattini, Acta Physica Polonica B 47, 1819 (2016).

[33] J. L. Friedman and B. F. Schutz, Astrophys. J. 200, 204 (1975).

[34] N. Andersson, Phys. Rev. D 86, 043002 (2012).

[35] N. Andersson, K. Dionysopoulou, I. Hawke, and G. L. Comer, Classical and Quantum Gravity 34, 125002 (2017).

[36] N. Andersson, I. Hawke, K. Dionysopoulou, and G. L. Comer, Classical and Quantum Gravity 34, 125003 (2017).

[37] N. Andersson and G. L. Comer, Living Reviews in Relativity 10, 1 (2007).

[38] D. Jou, G. Lebon, and J. Casas-Vzquez, Extended Irreversible Thermodynamics (Springer Netherlands, 2010).

[39] C. S. Lopez-Monsalvo and N. Andersson, Proceedings of the Royal Society of London Series A 467, 738 (2011).

[40] N. Andersson and C. S. Lopez-Monsalvo, Classical and Quantum Gravity 28, 195023 (2011).

[41] L. Samuelsson, C. S. Lopez-Monsalvo, N. Andersson, and G. L. Comer, General Relativity and Gravitation 42, 413 (2010).

[42] W. A. Hiscock and L. Lindblom, Phys. Rev. D 31, 725 (1985).

[43] L. Gavassino, M. Antonelli, and B. Haskell (2020), 2006.09843.

[44] L. Gavassino, M. Antonelli, and B. Haskell (2020), 2003.04609.

[45] L. E. Reichl, A Modern Course in Statistical Physics (Wiley-VCH, Weinheim, Germany, 2016).

[46] D. Montenegro and G. Torrieri, Phys. Rev. D 100, 056011 (2019).

[47] D. Montenegro and G. Torrieri (2020), 2004.10195.

[48] N. Andersson and G. L. Comer, Proceedings of the Royal Society of London Series A 466, 1373 (2010).

[49] L. Gavassino and M. Antonelli, Classical and Quantum Gravity 37, 025014 (2020). 